\title{
Probabilistic Independence Networks for Hidden Markov Probability Models
}

\author{
Padhraic Smyth \\ Department of Information and Computer Science, University of California at Irvine, \\ Irvine, CA 92697-3425 USA \\ and \\ Jet Propulsion Laboratory 525-3660, California Institute of Technology, \\ Pasadena, CA 91109 USA
}

David Heckerman

Microsoft Research, Redmond, WA 98052-6399 USA

Michael I. Jordan

Department of Brain and Cognitive Sciences, Massachusetts Institute of Technology, Cambridge, MA 02139 USA

Graphical techniques for modeling the dependencies of random variables have been explored in a variety of different areas, including statistics, statistical physics, artificial intelligence, speech recognition, image processing, and genetics. Formalisms for manipulating these models have been developed relatively independently in these research communities. In this paper we explore hidden Markov models (HMMs) and related structures within the general framework of probabilistic independence networks (PINs). The paper presents a self-contained review of the basic principles of PINs. It is shown that the well-known forward-backward (F-B) and Viterbi algorithms for HMMs are special cases of more general inference algorithms for arbitrary PINs. Furthermore, the existence of inference and estimation algorithms for more general graphical models provides a set of analysis tools for HMM practitioners who wish to explore a richer class of HMM structures. Examples of relatively complex models to handle sensor fusion and coarticulation in speech recognition are introduced and treated within the graphical model framework to illustrate the advantages of the general approach.

\section{Introduction}

For multivariate statistical modeling applications, such as hidden Markov modeling (HMM) for speech recognition, the identification and manipulation of relevant conditional independence assumptions can be useful for model building and analysis. There has recently been a considerable amount 
of work exploring the relationships between conditional independence in probability models and structural properties of related graphs. In particular, the separation properties of a graph can be directly related to conditional independence properties in a set of associated probability models.

The key point of this article is that the analysis and manipulation of generalized HMMs (more complex HMMs than the standard first-order model) can be facilitated by exploiting the relationship between probability models and graphs. The major advantages to be gained are in two areas:

- Model description. A graphical model provides a natural and intuitive medium for displaying dependencies that exist between random variables. In particular, the structure of the graphical model clarifies the conditional independencies in the associated probability models, allowing model assessment and revision.

- Computational efficiency. The graphical model is a powerful basis for specifying efficient algorithms for computing quantities of interest in the probability model (e.g., calculation of the probability of observed data given the model). These inference algorithms can be specified automatically once the initial structure of the graph is determined.

We will refer to both probability models and graphical models. Each consists of structure and parameters. The structure of the model consists of the specification of a set of conditional independence relations for the probability model or a set of (missing) edges in the graph for the graphical model. The parameters of both the probability and graphical models consist of the specification of the joint probability distribution: in factored form for the probability model and defined locally on the nodes of the graph for the graphical model. The inference problem is that of the calculation of posterior probabilities of variables of interest given observable data and a specification of the probabilistic model. The related task of maximum a posteriori (MAP) identification is the determination of the most likely state of a set of unobserved variables, given observed variables and the probabilistic model. The learning or estimation problem is that of determining the parameters (and possibly structure) of the probabilistic model from data.

This article reviews the applicability and utility of graphical modeling to HMMs and various extensions of HMMs. Section 2 introduces the basic notation for probability models and associated graph structures. Section 3 summarizes relevant results from the literature on probabilistic independence networks (PINs), in particular, the relationships that exist between separation in a graph and conditional independence in a probability model. Section 4 interprets the standard first-order HMM in terms of PINs. In Section 5 the standard algorithm for inference in a directed PIN is discussed and applied to the standard HMM in Section 6. A result of interest is that the forward-backward (F-B) and Viterbi algorithms are shown to be special cases of this inference algorithm. Section 7 shows that the inference algo- 
rithms for undirected PINs are essentially the same as those already discussed for directed PINs. Section 8 introduces more complex HMM structures for speech modeling and analyzes them using the graphical model framework. Section 9 reviews known estimation results for graphical models and discusses their potential implications for practical problems in the estimation of HMM structures, and Section 10 contains summary remarks.

\section{Notation and Background}

Let $\mathbf{U}=\left\{X_{1}, X_{2}, \ldots, X_{N}\right\}$ represent a set of discrete-valued random variables. For the purposes of this article we restrict our attention to discretevalued random variables; however, many of the results stated generalize directly to continuous and mixed sets of random variables (Lauritzen and Wermuth 1989; Whittaker 1990). Let lowercase $x_{i}$ denote one of the values of variable $X_{i}$ : the notation $\sum_{x_{1}}$ is taken to mean the sum over all possible values of $X_{1}$. Let $p\left(x_{i}\right)$ be shorthand for the particular probability $p\left(X_{i}=x_{i}\right)$, whereas $p\left(X_{i}\right)$ represents the probability function for $X_{i}$ (a table of values, since $X_{i}$ is assumed discrete), $1 \leq i \leq N$. The full joint distribution function is $p(\mathbf{U})=p\left(X_{1}, X_{2}, \ldots, X_{N}\right)$, and $p(\mathbf{u})=\left(x_{1}, x_{2}, \ldots, x_{N}\right)$ denotes a particular value assignment for $\mathbf{U}$. Note that this full joint distribution $p(\mathbf{U})=p\left(X_{1}, X_{2}, \ldots, X_{N}\right)$ provides all the possible information one needs to calculate any marginal or conditional probability of interest among subsets of $\mathbf{U}$.

If $A, B$, and $C$ are disjoint sets of random variables, the conditional independence relation $A \perp B \mid C$ is defined such that $A$ is independent of $B$ given $C$, that is, $p(A, B \mid C)=p(A \mid C) p(B \mid C)$. Conditional independence is symmetric. Note also that marginal independence (no conditioning) does not in general imply conditional independence, nor does conditional independence in general imply marginal independence (Whittaker 1990).

With any set of random variables $\mathbf{U}$ we can associate a graph $G$ defined as $G=(V, E) . V$ denotes the set of vertices or nodes of the graph such that there is a one-to-one mapping between the nodes in the graph and the random variables, that is, $V=\left\{X_{1}, X_{2}, \ldots, X_{N}\right\}$. E denotes the set of edges, $\{e(i, j)\}$, where $i$ and $j$ are shorthand for the nodes $X_{i}$ and $X_{j}, 1 \leq i, j \leq N$. Edges of the form $e(i, i)$ are not of interest and thus are not allowed in the graphs discussed in this article.

An edge may be directed or undirected. Our convention is that a directed edge $e(i, j)$ is directed from node $i$ to node $j$, in which case we sometimes say that $i$ is a parent of its child $j$. An ancestor of node $i$ is a node that has as a child either $i$ or another ancestor of $i$. A subset of nodes $A$ is an ancestral set if it contains its own ancestors. A descendant of $i$ is either a child of $i$ or a child of a descendant of $i$.

Two nodes $i$ and $j$ are adjacent in $G$ if $E$ contains the undirected or directed edge $e(i, j)$. An undirected path is a sequence of distinct nodes $\{1, \ldots, m\}$ such that there exists an undirected or directed edge for each pair of nodes 
$\{l, l+1\}$ on the path. A directed path is a sequence of distinct nodes $\{1, \ldots, m\}$ such that there exists a directed edge for each pair of nodes $\{l, l+1\}$ on the path. A graph is singly connected if there exists only one undirected path between any two nodes in the graph. An (un)directed cycle is a path such that the beginning and ending nodes on the (un)directed path are the same.

If $E$ contains only undirected edges, then the graph $G$ is an undirected graph (UG). If $E$ contains only directed edges then the graph $G$ is a directed graph (DG).

Two important classes of graphs for modeling probability distributions that we consider in this paper are UGs and acyclic directed graphs (ADGs)directed graphs having no directed cycles. We note in passing that there exists a theory for graphical independence models involving both directed and undirected edges (chain graphs, Whittaker 1990), but these are not discussed here.

For a UG $G$, a subset of nodes $C$ separates two other subsets of nodes $A$ and $B$ if every path joining every pair of nodes $i \in A$ and $j \in B$ contains at least one node from $C$. For ADGs, analogous but somewhat more complicated separation properties exist.

A graph $G$ is complete if there are edges between all pairs of nodes. A cycle in an undirected graph is chordless if none other than successive pairs of nodes in the cycle are adjacent. An undirected graph $G$ is triangulated if and only if the only chordless cycles in the graph contain no more than three nodes. Thus, if one can find a chordless cycle of length four or more, $G$ is not triangulated. A clique in an undirected graph $G$ is a subgraph of $G$ that is complete. A clique tree for $G$ is a tree of cliques such that there is a one-to-one correspondence between the cliques of $G$ and the nodes of the tree.

\section{Probabilistic Independence Networks}

We briefly review the relation between a probability model $p(\mathbf{U})=p\left(X_{1}\right.$, $\left.\ldots, X_{N}\right)$ and a probabilistic independence network structure $G=(V, E)$ where the vertices $V$ are in one-to-one correspondence with the random variables in $\mathbf{U}$. (The results in this section are largely summarized versions of material in Pearl 1988 and Whittaker 1990.)

A PIN structure $G$ is a graphical statement of a set of conditional independence relations for a set of random variables $\mathbf{U}$. Absence of an edge $e(i, j)$ in $G$ implies some independence relation between $X_{i}$ and $X_{j}$. Thus, a PIN structure $G$ is a particular way of specifying the independence relationships present in the probability model $p(\mathbf{U})$. We say that $G$ implies a set of probability models $p(\mathbf{U})$, denoted as $\mathcal{P}_{G}$, that is, $p(\mathbf{U}) \in \mathcal{P}_{G}$. In the reverse direction, a particular model $p(\mathbf{U})$ embodies a particular set of conditional independence assumptions that may or may not be representable in a consistent graphical form. One can derive all of the conditional independence 


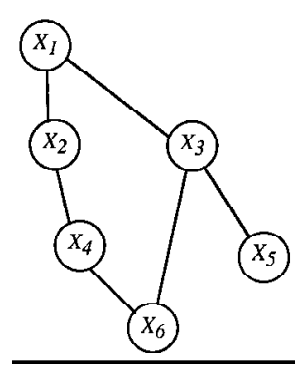

Figure 1: An example of a UPIN structure $G$ that captures a particular set of conditional independence relationships among the set of variables $\left\{X_{1}, \ldots, X_{6}\right\}$-for example, $X_{5} \perp\left\{X_{1}, X_{2}, X_{4}, X_{6}\right\} \mid\left\{X_{3}\right\}$.

properties and inference algorithms of interest for $\mathbf{U}$ without reference to graphical models. However, as has been emphasized in the statistical and artificial intelligence literature, and as reiterated in this article in the context of HMMs, there are distinct advantages to be gained from using the graphical formalism.

3.1 Undirected Probabilistic Independence Networks (UPINs). A UPIN is composed of both a UPIN structure and UPIN parameters. A UPIN structure specifies a set of conditional independence relations for a probability model in the form of an undirected graph. UPIN parameters consist of numerical specifications of a particular probability model consistent with the UPIN structure. Terms used in the literature to describe UPINs of one form or another include Markov random fields (Isham 1981; Geman and Geman 1984), Markov networks (Pearl 1988), Boltzmann machines (Hinton and Sejnowski 1986), and log-linear models (Bishop et al. 1973).

3.1.1 Conditional Independence Semantics of UPIN Structures. Let $A, B$, and $S$ be any disjoint subsets of nodes in an undirected graph $G$. $G$ is a UPIN structure for $p(\mathbf{U})$ if for any $A, B$, and $S$ such that $S$ separates $A$ and $B$ in $G$, the conditional independence relation $A \perp B \mid S$ holds in $p(\mathbf{U})$. The set of all conditional independence relations implied by separation in $G$ constitutes the (global) Markov properties of G. Figure 1 shows a simple example of a UPIN structure for six variables.

Thus, separation in the UPIN structure implies conditional independence in the probability model; i.e., it constrains $p(\mathbf{U})$ to belong to a set of probability models $\mathcal{P}_{G}$ that obey the Markov properties of the graph. Note that a complete UG is trivially a UPIN structure for any $p(\mathbf{U})$ in the sense that 
there are no constraints on $p(\mathbf{U}) . G$ is a perfect undirected map for $p$ if $G$ is a UPIN structure for $p(\mathbf{U})$ and all the conditional independence relations present in $p(\mathbf{U})$ are represented by separation in $G$. For many probability models $p$ there are no perfect undirected maps. A weaker condition is that a UPIN structure $G$ is minimal for a probability model $p(\mathbf{U})$ if the removal of any edge from $G$ implies an independence relation not present in the model $p(\mathbf{U})$; that is, the structure without the edge is no longer a UPIN structure for $p(\mathbf{U})$. Minimality is not equivalent to perfection (for UPIN structures) since, for example, there exist probability models with independencies that cannot be represented as UPINs except for the complete UPIN structure. For example, consider that $X$ and $Y$ are marginally independent but conditionally dependent given $Z$ (e.g., $X$ and $Y$ are two independent causal variables with a common effect $Z$ ). In this case the complete graph is the minimal UPIN structure for $\{X, Y, Z\}$, but it is not perfect because of the presence of an edge between $X$ and $Y$.

\subsubsection{Probability Functions on UPIN structures. Given a UPIN structure} $G$, the joint probability distribution for $\mathbf{U}$ can be expressed as a simple factorization:

$$
p(\mathbf{u})=p\left(x_{1}, \ldots, x_{N}\right)=\prod_{V_{C}} a_{C}\left(x_{C}\right)
$$

where $V_{C}$ is the set of cliques of $G, x_{C}$ represents an assignment of values to the variables in a particular clique $C$, and the $a_{C}\left(x_{C}\right)$ are non-negative clique functions. (The domain of each $a_{C}\left(x_{C}\right)$ is the set of possible assignments of values to the variables in the clique $C$, and the range of $a_{C}\left(x_{C}\right)$ is the semiinfinite interval $[0, \infty)$.) The set of clique functions associated with a UPIN structure provides the numerical parameterization of the UPIN.

A UPIN is equivalent to a Markov random field (Isham 1981). In the Markov random field literature the clique functions are generally referred to as potential functions. A related terminology, used in the context of the Boltzmann machine (Hinton and Sejnowski 1986), is that of energy function. The exponential of the negative energy of a configuration is a Boltzmann factor. Scaling each Boltzmann factor by the sum across Boltzmann factors (the partition function) yields a factorization of the joint density (the Boltzmann distribution), that is, a product of clique functions. ${ }^{1}$ The advantage of defining clique functions directly rather than in terms of the exponential of an energy function is that the range of the clique functions can be allowed

${ }^{1}$ A Boltzmann machine is a special case of a UPIN in which the clique functions can be decomposed into products of factors associated with pairs of variables. If the Boltzmann machine is augmented to include "higher-order" energy terms, one for each clique in the graph, then we have a general Markov random field or UPIN, restricted to positive probability distributions due to the exponential form of the clique functions. 


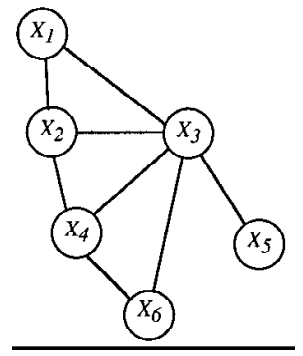

Figure 2: A triangulated version of the UPIN structure G from Figure 1.

to contain zero. Thus equation 3.1 can represent configurations of variables having zero probability.

A model $p$ is said to be decomposable if it has a minimal UPIN structure $G$ that is triangulated (see Fig. 2). A UPIN structure $G$ is decomposable if $G$ is triangulated. For the special case of decomposable models, $G$ can be converted to a junction tree, which is a tree of cliques of $G$ arranged such that the cliques satisfy the running intersection property, namely, that each node in $G$ that appears in any two different cliques also appears in all cliques on the undirected path between these two cliques. Associated with each edge in the junction tree is a separator $S$, such that $S$ contains the variables in the intersection of the two cliques that it links. Given a junction tree representation, one can factorize $p(\mathbf{U})$ as the product of clique marginals over separator marginals (Pearl 1988):

$$
p(\mathbf{u})=\frac{\prod_{C \in V_{C}} p\left(x_{C}\right)}{\prod_{S \in V_{S}} p\left(x_{S}\right)},
$$

where $p\left(x_{C}\right)$ and $p\left(x_{S}\right)$ are the marginal (joint) distributions for the variables in clique $C$ and separator $S$, respectively, and $V_{C}$ and $V_{S}$ are the set of cliques and separators in the junction tree.

This product representation is central to the results in the rest of the article. It is the basis of the fact that globally consistent probability calculations on $\mathbf{U}$ can be carried out in a purely local manner. The mechanics of these local calculations will be described later. At this point it is sufficient to note that the complexity of the local inference algorithms scales as the sum of the sizes of the clique state-spaces (where a clique state-space is equal to the product over each variable in the clique of the number of states of each variable). Thus, local clique updating can make probability calculations on U much more tractable than using "brute force" inference if the model decomposes into relatively small cliques. 
Many probability models of interest may not be decomposable. However, we can define a decomposable cover $G^{\prime}$ for $p$ such that $G^{\prime}$ is a triangulated, but not necessarily minimal, UPIN structure for $p$. Since any UPIN $G$ can be triangulated simply by the addition of the appropriate edges, one can always identify at least one decomposable cover $G^{\prime}$. However, a decomposable cover may not be minimal in that it can contain edges that obscure certain independencies in the model $p$; for example, the complete graph is a decomposable cover for all possible probability models $p$. For efficient inference, the goal is to find a decomposable cover $G^{\prime}$ such that $G^{\prime}$ contains as few extra edges as possible over the original UPIN structure $G$. Later we discuss a specific algorithm for finding decomposable covers for arbitrary PIN structures. All singly connected UPIN structures imply probability models $\mathcal{P}_{\mathcal{G}}$ that are decomposable.

Note that given a particular probability model $p$ and a UPIN $G$ for $p$, the process of adding extra edges to $G$ to create a decomposable cover does not change the underlying probability model $p$; that is, the added edges are a convenience for manipulating the graphical representation, but the underlying numerical probability specifications remain unchanged.

An important point is that decomposable covers have the running intersection property and thus can be factored as in equation 3.2. Thus local clique updating is also possible with nondecomposable models by this conversion. Once again, the complexity of such local inference scales with the sum of the size of the clique state-spaces in the decomposable cover.

In summary, any UPIN structure can be converted to a junction tree permitting inference calculations to be carried out purely locally on cliques.

3.2 Directed Probabilistic Independence Networks (DPINs). A DPIN is composed of both a DPIN structure and DPIN parameters. A DPIN structure specifies a set of conditional independence relations for a probability model in the form of a directed graph. DPIN parameters consist of numerical specifications of a particular probability model consistent with the DPIN structure. DPINs are referred to in the literature using different names, including Bayes network, belief network, recursive graphical model, causal (belief) network, and probabilistic (causal) network.

\subsubsection{Conditional Independence Semantics of DPIN Structures. A DPIN} structure is an ADG $G^{D}=(V, E)$ where there is a one-to-one correspondence between $V$ and the elements of the set of random variables $\mathbf{U}=$ $\left\{X_{1}, \ldots, X_{N}\right\}$.

It is convenient to define the moral graph $G^{M}$ of $G^{D}$ as the undirected graph obtained from $G^{D}$ by placing undirected edges between all nonadjacent parents of each node and then dropping the directions from the remaining directed edges (see Fig. 3b for an example). The term moral was coined to denote the "marrying" of "unmarried" (nonadjacent) parents. The motivation behind this procedure will become clear when we discuss the differ- 


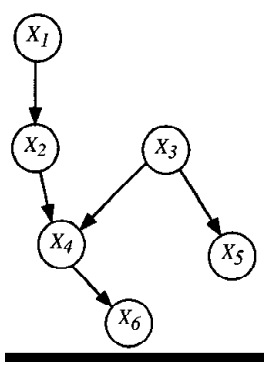

(a)

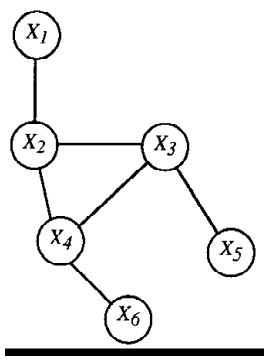

(b)

Figure 3: (a) A DPIN structure $G^{D}$ that captures a set of independence relationships among the set $\left\{X_{1}, \ldots, X_{5}\right\}$-for example, $X_{4} \perp X_{1} \mid X_{2}$. (b) The moral graph $G^{M}$ for $G^{D}$, where the parents of $X_{4}$ have been linked.

ences between DPINs and UPINs in Section 3.3. We shall also see that this conversion of a DPIN into a UPIN is a convenient way to solve DPIN inference problems by "transforming" the problem into an undirected graphical setting and taking advantage of the general theory available for undirected graphical models.

We can now define a DPIN as follows. Let $A, B$, and $S$ be any disjoint subsets of nodes in $G^{D}$. $G^{D}$ is a DPIN structure for $p(\mathbf{U})$ if for any $A, B$, and $S$ such that $S$ separates $A$ and $B$ in $G^{D}$, the conditional independence relation $A \perp B \mid S$ holds in $p(\mathbf{U})$. This is the same definition as for a UPIN structure except that separation has a more complex interpretation in the directed context: $S$ separates $A$ from $B$ in a directed graph if $S$ separates $A$ from $B$ in the moral (undirected) graph of the smallest ancestral set containing $A, B$, and $S$ (Lauritzen et al. 1990). It can be shown that this definition of a DPIN structure is equivalent to the more intuitive statement that given the values of its parents, a variable $X_{i}$ is independent of all other nodes in the directed graph except for its descendants. 
Thus, as with a UPIN structure, the DPIN structure implies certain conditional independence relations, which in turn imply a set of probability models $p \in \mathcal{P}_{G^{D}}$. Figure 3a contains a simple example of a DPIN structure.

There are many possible DPIN structures consistent with a particular probability model $p(\mathbf{U})$, potentially containing extra edges that hide true conditional independence relations. Thus, one can define minimal DPIN structures for $p(\mathbf{U})$ in a manner exactly equivalent to that of UPIN structures: Deletion of an edge in a minimal DPIN structure $G^{D}$ implies an independence relation that does not hold in $p(\mathbf{U}) \in \mathcal{P}_{G^{D}}$. Similarly, $G^{D}$ is a perfect DPIN structure $G$ for $p(\mathbf{U})$ if $G^{D}$ is a DPIN structure for $p(\mathbf{U})$ and all the conditional independence relations present in $p(\mathbf{U})$ are represented by separation in $G^{D}$. As with UPIN structures, minimal does not imply perfect for DPIN structures. For example, consider the independence relations $X_{1} \perp X_{4} \mid\left\{X_{2}, X_{3}\right\}$ and $X_{2} \perp X_{3} \mid\left\{X_{1}, X_{4}\right\}$ : the minimal DPIN structure contains an edge from $X_{3}$ to $X_{2}$ (see Fig. $4 b$ ). A complete ADG is trivially a DPIN structure for any probability model $p(\mathbf{U})$.

3.2.2 Probability Functions on DPINs. A basic property of a DPIN structure is that it implies a direct factorization of the joint probability distribution $p(\mathbf{U})$ :

$$
p(\mathbf{u})=\prod_{i=1}^{N} p\left(x_{i} \mid p a\left(x_{i}\right)\right),
$$

where $p a\left(x_{i}\right)$ denotes a value assignment for the parents of $X_{i}$. A probability model $p$ can be written in this factored form in a trivial manner by the conditioning rule. Note that a directed graph containing directed cycles does not necessarily yield such a factorization, hence the use of ADGs.

\subsection{Differences between Directed and Undirected Graphical Repre-} sentations. It is an important point that directed and undirected graphs possess different conditional independence semantics. There are common conditional independence relations that have perfect DPIN structures but no perfect UPIN structures, and vice versa (see Figure 4 for examples).

Does a DPIN structure have the same Markov properties as the UPIN structure obtained by dropping all the directions on the edges in the DPIN structure? The answer is yes if and only if the DPIN structure contains no subgraphs where a node has two or more nonadjacent parents (Whittaker 1990; Pearl et al. 1990). In general, it can be shown that if a UPIN structure G for $p$ is decomposable (triangulated), then it has the same Markov properties as some DPIN structure for $p$.

On a more practical level, DPIN structures are frequently used to encode causal information, that is, to represent the belief formally that $X_{i}$ precedes $X_{j}$ in some causal sense (e.g., temporally). DPINs have found application in causal modeling in applied statistics and artificial intelligence. Their popularity in these fields stems from the fact that the joint probability model can 


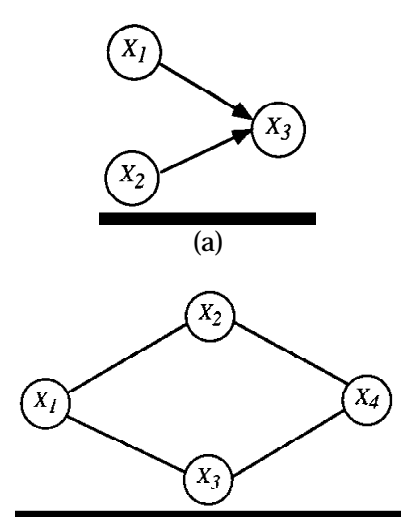

(b)

Figure 4: (a) The DPIN structure to encode the fact that $X_{3}$ depends on $X_{1}$ and $X_{2}$ but $X_{1} \perp X_{2}$. For example, consider that $X_{1}$ and $X_{2}$ are two independent coin flips and that $X_{3}$ is a bell that rings when the flips are the same. There is no perfect UPIN structure that can encode these dependence relationships. (b) A UPIN structure that encodes $X_{1} \perp X_{4} \mid\left\{X_{2}, X_{3}\right\}$ and $X_{2} \perp X_{3} \mid\left\{X_{1}, X_{4}\right\}$. There is no perfect DPIN structure that can encode these dependencies.

be specified directly by equation 3.3, that is, by the specification of conditional probability tables or functions (Spiegelhalter el al. 1991). In contrast, UPINs must be specified in terms of clique functions (as in equation 3.1), which may not be as easy to work with (cf. Geman and Geman 1984, Modestino and Zhang 1992, and Vandermeulen et al. 1994 for examples of ad hoc design of clique functions in image analysis). UPINs are more frequently used in problems such as image analysis and statistical physics where associations are thought to be correlational rather than causal.

3.4 From DPINs to (Decomposable) UPINs. The moral UPIN structure $G^{M}$ (obtained from the DPIN structure $G^{D}$ ) does not imply any new independence relations that are not present in $G^{D}$. As with triangulation, however, the additional edges may obscure conditional independence relations implicit in the numeric specification of the original probability model $p$ associated with the DPIN structure $G^{D}$. Furthermore, $G^{M}$ may not be triangulated (decomposable). By the addition of appropriate edges, the moral graph can be converted to a (nonunique) triangulated graph $G^{\prime}$, namely, a decomposable cover for $G^{M}$. In this manner, for any probability model $p$ for which $G^{D}$ is a DPIN structure, one can construct a decomposable cover $G^{\prime}$ for $p$.

This mapping from DPIN structures to UPIN structures was first discussed in the context of efficient inference algorithms by Lauritzen and 
Spiegelhalter (1988). The advantage of this mapping derives from the fact that analysis and manipulation of the resulting UPIN are considerably more direct than dealing with the original DPIN. Furthermore, it has been shown that many of the inference algorithms for DPINs are in fact special cases of inference algorithms for UPINs and can be considerably less efficient (Shachter et al. 1994).

\section{Modeling HMMs as PINs}

4.1 PINs for HMMs. In hidden Markov modeling problems (Baum and Petrie 1966; Poritz 1988; Rabiner 1989; Huang et al. 1990; Elliott et al. 1995) we are interested in the set of random variables $\mathbf{U}=\left\{\mathrm{H}_{1}, \mathrm{O}_{1}, \mathrm{H}_{2}, \mathrm{O}_{2}, \ldots\right.$, $\left.H_{N-1}, O_{N-1}, H_{N}, O_{N}\right\}$, where $H_{i}$ is a discrete-valued hidden variable at index $i$, and $O_{i}$ is the corresponding discrete-valued observed variable at index $i$, $1 \leq i \leq N$. (The results here can be directly extended to continuous-valued observables.) The index $i$ denotes a sequence from 1 to $N$, for example, discrete time steps. Note that $O_{i}$ is considered univariate for convenience: the extension to the multivariate case with $d$ observables is straightforward but is omitted here for simplicity since it does not illuminate the conditional independence relationships in the HMM.

The well-known simple first-order HMM obeys the following two conditional independence relations:

$$
H_{i} \perp\left\{H_{1}, O_{1}, \ldots, H_{i-2}, O_{i-2}, O_{i-1}\right\} \mid H_{i-1}, \quad 3 \leq i \leq N
$$

and

$$
O_{i} \perp\left\{H_{1}, O_{1}, \ldots, H_{i-1}, O_{i-1}\right\} \mid H_{i}, \quad 2 \leq i \leq N
$$

We will refer to this "first-order" hidden Markov probability model as $\operatorname{HMM}(1,1)$ : the notation $\operatorname{HMM}(K, J)$ is defined such that the hidden state of the model is represented via the conjoined configuration of $J$ underlying random variables and such that the model has state memory of depth $K$. The notation will be clearer in later sections when we discuss specific examples with $K, J>1$.

Construction of a PIN for $\operatorname{HMM}(1,1)$ is simple. In the undirected case, assumption 1 requires that each state $H_{i}$ is connected to $H_{i-1}$ only from the set $\left\{H_{1}, O_{1}, \ldots, H_{i-2}, O_{i-2}, H_{i-1}, O_{i-1}\right\}$. Assumption 2 requires that $O_{i}$ is connected only to $H_{i}$. The resulting UPIN structure for $\operatorname{HMM}(1,1)$ is shown in Figure 5a. This graph is singly connected and thus implies a decomposable probability model $p$ for $\operatorname{HMM}(1,1)$, where the cliques are of the form $\left\{H_{i}, O_{i}\right\}$ and $\left\{H_{i-1}, H_{i}\right\}$ (see Fig. 5b). In Section 5 we will see how the joint probability function can be expressed as a product function on the junction tree, thus leading to a junction tree definition of the familiar F-B and Viterbi inference algorithms. 


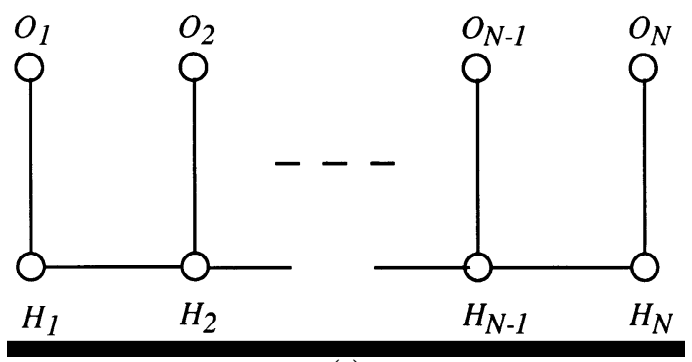

(a)

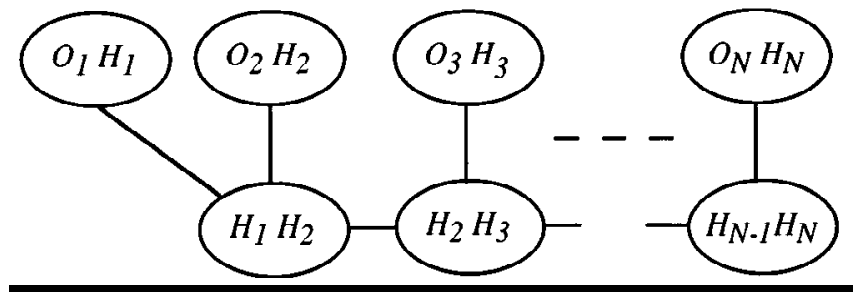

(b)

Figure 5: (a) PIN structure for $\operatorname{HMM}(1,1)$. (b) A corresponding junction tree.

For the directed case, the connectivity for the DPIN structure is the same. It is natural to choose the directions on the edges between $H_{i-1}$ and $H_{i}$ as going from $i-1$ to $i$ (although the reverse direction could also be chosen without changing the Markov properties of the graph). The directions on the edges between $H_{i}$ and $O_{i}$ must be chosen as going from $H_{i}$ to $O_{i}$ rather than in the reverse direction (see Figure 6a). In reverse (see Fig. 6b) the arrows would imply that $O_{i}$ is marginally independent of $H_{i-1}$, which is not true in the $\operatorname{HMM}(1,1)$ probability model. The proper direction for the edges implies the correct relation, namely, that $O_{i}$ is conditionally independent of $H_{i-1}$ given $H_{i}$.

The DPIN structure for $\operatorname{HMM}(1,1)$ does not possess a subgraph with nonadjacent parents. As stated earlier, this implies that the implied independence properties of the DPIN structure are the same as those of the corresponding UPIN structure obtained by dropping the directions from the edges in the DPIN structure, and thus they both result in the same junction tree structure (see Fig. 5b). Thus, for the $\operatorname{HMM}(1,1)$ probability model, the minimal directed and undirected graphs possess the same Markov properties; they imply the same conditional independence relations. Furthermore, both PIN structures are perfect maps for the directed and undirected cases, respectively. 


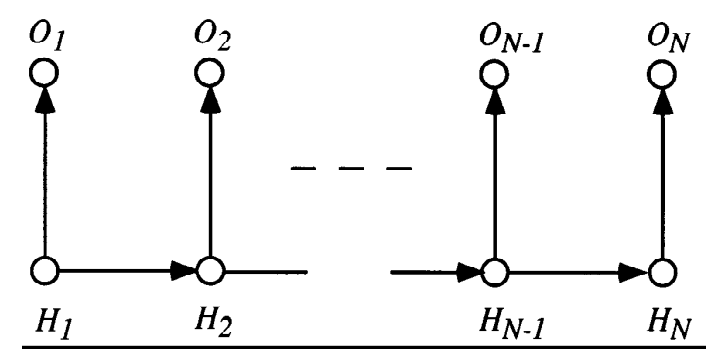

(a)

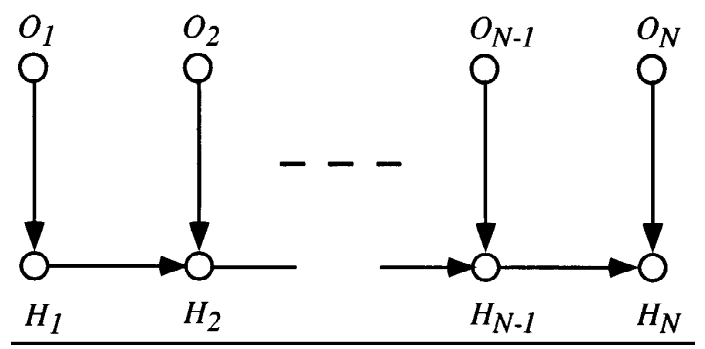

(b)

Figure 6: DPIN structures for $\operatorname{HMM}(1,1)$. (a) The DPIN structure for the $\operatorname{HMM}(1,1)$ probability model. (b) A DPIN structure that is not a DPIN structure for the $\operatorname{HMM}(1,1)$ probability model.

4.2 Inference and MAP Problems in HMMs. In the context of HMMs, the most common inference problem is the calculation of the likelihood of the observed evidence given the model, that is, $p\left(o_{1}, \ldots, o_{N} \mid\right.$ model $)$, where the $o_{1}, \ldots, o_{N}$ denote observed values for $O_{1}, \ldots, O_{N}$. (In this section we will assume that we are dealing with one particular model where the structure and parameters have already been determined, and thus we will not explicitly indicate conditioning on the model.) The "brute force" method for obtaining this probability would be to sum out the unobserved state variables from the full joint probability distribution:

$$
p\left(o_{1}, \ldots, o_{N}\right)=\sum_{h_{1}, \ldots, h_{N}} p\left(H_{1}, o_{1}, \ldots, H_{N}, o_{N}\right)
$$

where $h_{i}$ denotes the possible values of hidden variable $H_{i}$.

In general, both of these computations scale as $m^{N}$ where $m$ is the number of states for each hidden variable. In practice, the F-B algorithm (Poritz 1988; Rabiner 1989) can perform these inference calculations with much lower complexity, namely, $\mathrm{Nm}^{2}$. The likelihood of the observed evidence 
can be obtained with the forward step of the F-B algorithm: calculation of the state posterior probabilities requires both forward and backward steps. The F-B algorithm relies on a factorization of the joint probability function to obtain locally recursive methods. One of the key points in this article is that the graphical modeling approach provides an automatic method for determining such local efficient factorizations, for an arbitrary probabilistic model, if efficient factorizations exist given the conditional independence (CI) relations specified in the model.

The MAP identification problem in the context of HMMs involves identifying the most likely hidden state sequence given the observed evidence. Just as with the inference problem, the Viterbi algorithm provides an efficient, locally recursive method for solving this problem with complexity $\mathrm{Nm}^{2}$, and again, as with the inference problem, the graphical modeling approach provides an automatic technique for determining efficient solutions to the MAP problem for arbitrary models, if an efficient solution is possible given the structure of the model.

\section{Inference and MAP Algorithms for DPINs}

Inference and MAP algorithms for DPINs and UPINS are quite similar: the UPIN case involves some subtleties not encountered in DPINs, and so discussion of UPIN inference and MAP algorithms is deferred until Section 7. The inference algorithm for DPINs (developed by Jensen et al. 1990, and hereafter referred to as the JLO algorithm) is a descendant of an inference algorithm first described by Lauritzen and Spiegelhalter (1988). The JLO algorithm applies to discrete-valued variables: extensions to the JLO algorithm for gaussian and gaussian-mixture distributions are discussed in Lauritzen and Wermuth (1989). A closely related algorithm to the JLO algorithm, developed by Dawid (1992a), solves the MAP identification problem with the same time complexity as the JLO inference algorithm.

We show that the JLO and Dawid algorithms are strict generalizations of the well-known F-B and Viterbi algorithms for $\operatorname{HMM}(1,1)$ in that they can be applied to arbitrarily complex graph structures (and thus a large family of probabilistic models beyond $\operatorname{HMM}(1,1))$ and handle missing values, partial inference, and so forth in a straightforward manner.

There are many variations on the basic JLO and Dawid algorithms. For example, Pearl (1988) describes related versions of these algorithms in his early work. However, it can be shown (Shachter et al. 1994) that all known exact algorithms for inference on DPINs are equivalent at some level to the JLO and Dawid algorithms. Thus, it is sufficient to consider the JLO and Dawid algorithms in our discussion as they subsume other graphical inference algorithms. ${ }^{2}$

\footnotetext{
${ }^{2}$ An alternative set of computational formalisms is provided by the statistical physics
} 
The JLO and Dawid algorithms operate as a two-step process:

1. The construction step. This involves a series of substeps where the original directed graph is moralized and triangulated, a junction tree is formed, and the junction tree is initialized.

2. The propagation step. The junction tree is used in a local messagepassing manner to propagate the effects of observed evidence, that is, to solve the inference and MAP problems.

The first step is carried out only once for a given graph. The second (propagation) step is carried out each time a new inference for the given graph is requested.

5.1 The Construction Step of the JLO Algorithm: From DPIN Structures to Junction Trees. We illustrate the construction step of the JLO algorithm using the simple DPIN structure, $G^{D}$, over discrete variables $\mathbf{U}=$ $\left\{X_{1}, \ldots, X_{6}\right\}$ shown in Figure $7 \mathrm{a}$. The JLO algorithm first constructs the moral graph $G^{M}$ (see Fig. 7b). It then triangulates the moral graph $G^{M}$ to obtain a decomposable cover $G^{\prime}$ (see Fig. 7c). The algorithm operates in a simple, greedy manner based on the fact that a graph is triangulated if and only if all of its nodes can be eliminated, where a node can be eliminated whenever all of its neighbors are pairwise-linked. Whenever a node is eliminated, it and its neighbors define a clique in the junction tree that is eventually constructed. Thus, we can triangulate a graph and generate the cliques for the junction tree by eliminating nodes in some order, adding links if necessary. If no node can be eliminated without adding links, then we choose the node that can be eliminated by adding the links that yield the clique with the smallest state-space.

After triangulation, the JLO algorithm constructs a junction tree from $G^{\prime}$ (i.e., a clique tree satisfying the running intersection property). The junction tree construction is based on the following fact: Define the weight of a link

literature, where undirected graphical models in the form of chains, trees, lattices, and "decorated" variations on chains and trees have been studied for many years (see, e.g., Itzykson and Drouffé 1991). The general methods developed there, notably the transfer matrix formalism (e.g., Morgenstern and Binder 1983), support exact calculations on general undirected graphs. The transfer matrix recursions and the calculations in the JLO algorithm are closely related, and a reasonable hypothesis is that they are equivalent formalisms. (The question does not appear to have been studied in the general case, although see Stolorz 1994 and Saul and Jordan 1995 for special cases.) The appeal of the JLO framework, in the context of this earlier literature on exact calculations, is the link that it provides to conditional probability models (i.e., directed graphs) and the focus on a particular data structure- the junction tree-as the generic data structure underlying exact calculations. This does not, of course, diminish the potential importance of statistical physics methodology in graphical modeling applications. One area where there is clearly much to be gained from links to statistical physics is the area of approximate calculations, where a wide variety of methods are available (see, e.g., Swendsen and Wang 1987). 


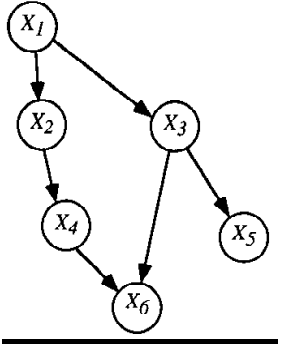

(a)

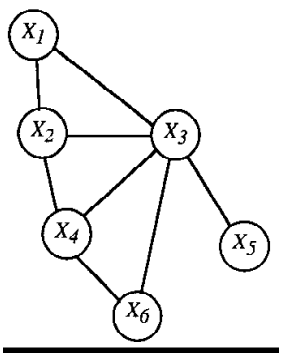

(c)

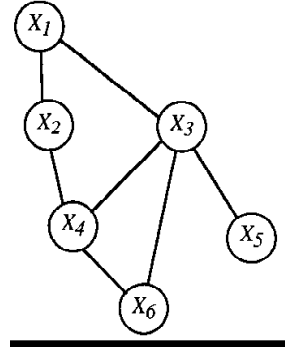

(b)

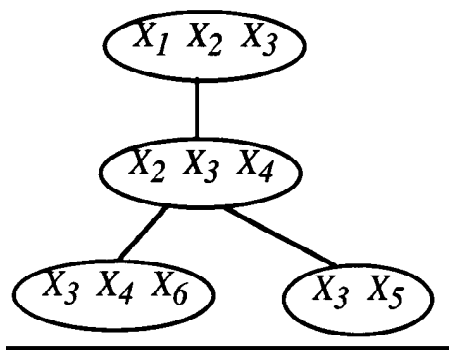

(d)

Figure 7: (a) A simple DPIN structure $G^{D}$. (b) The corresponding (undirected) moral graph $G^{M}$. (c) The corresponding triangulated graph $G^{\prime}$. (d) The corresponding junction tree.

between two cliques as the number of variables in their intersection. Then a tree of cliques will satisfy the running intersection property if and only if it is a spanning tree of maximal weight. Thus, the JLO algorithm constructs a junction tree by choosing successively a link of maximal weight unless it creates a cycle. The junction tree constructed from the cliques defined by the DPIN structure triangulation in Figure 7c is shown in Figure 7d.

The worst-case complexity is $O\left(N^{3}\right)$ for the triangulation heuristic and $O\left(N^{2} \log N\right)$ for the maximal spanning tree portion of the algorithm. This construction step is carried out only once as an initial step to convert the original graph to a junction tree representation.

5.2 Initializing the Potential Functions in the Junction Tree. The next step is to take the numeric probability specifications as defined on the directed graph $G^{D}$ (see equation 3.3) and convert this information into the general form for a junction tree representation of $p$ (see equation 3.2). This is achieved by noting that each variable $X_{i}$ is contained in at least one clique in the junction tree. Assign each $X_{i}$ to just one such clique, and for each clique 
define the potential function $a_{C}(C)$ to be either the product of $p\left(X_{i} \mid p a\left(X_{i}\right)\right)$ over all $X_{i}$ assigned to clique $C$, or 1 if no variables are assigned to that clique. Define the separator potentials (in equation 3.2) to be 1 initially.

In the section that follows, we describe the general JLO algorithm for propagating messages through the junction tree to achieve globally consistent probability calculations. At this point it is sufficient to know that a schedule of local message passing can be defined that converges to a globally consistent marginal representation for $p$; that is, the potential on any clique or separator is the marginal for that clique or separator (the joint probability function). Thus, via local message passing, one can go from the initial potential representation defined above to a marginal representation:

$$
p(\mathbf{u})=\frac{\prod_{C \in V_{C}} p\left(x_{C}\right)}{\prod_{S \in V_{S}} p\left(x_{S}\right)} .
$$

At this point the junction tree is initialized. This operation in itself is not that useful; of more interest is the ability to propagate information through the graph given some observed data and the initialized junction tree (e.g., to calculate the posterior distributions of some variables of interest).

From this point onward we will implicitly assume that the junction tree has been initialized as described so that the potential functions are the local marginals.

\subsection{Local Message Propagation in Junction Trees Using the JLO Algo-} rithm. In general $p(\mathbf{U})$ can be expressed as

$$
p(\mathbf{u})=\frac{\prod_{C \in V_{C}} a_{C}\left(x_{C}\right)}{\prod_{S \in V_{S}} b_{S}\left(x_{S}\right)},
$$

where the $a_{C}$ and $b_{S}$ are nonnegative potential functions (the potential functions could be the initial marginals described above, for example). Note that this representation is a generalization of the representations for $p(\mathbf{u})$ given by equations 3.1 and 3.2. $K=\left(\left\{a_{C}: C \in V_{C}\right\},\left\{b_{S}: S \in S_{C}\right\}\right)$ is a representation for $p(\mathbf{U})$. A factorizable function $p(\mathbf{U})$ can admit many different representations, that is, many different sets of clique and separator functions that satisfy equation 5.2 given a particular $p(\mathbf{U})$.

The JLO algorithm carries out globally consistent probability calculations via local message passing on the junction tree; probability information is passed between neighboring cliques, and clique and separator potentials are updated based on this local information. A key point is that the cliques and separators are updated in a fashion that ensures that at all times $K$ is a representation for $p(\mathbf{U})$; in other words, equation 5.2 holds at all times. Eventually the propagation converges to the marginal representation given the initial model and the observed evidence. 
The message passing proceeds as follows: We can define a flow from clique $C_{i}$ to $C_{j}$ in the following manner where $C_{i}$ and $C_{j}$ are two cliques adjacent in the junction tree. Let $S_{k}$ be the separator for these two cliques. Define

$$
b_{S_{k}}^{*}\left(x_{S_{k}}\right)=\sum_{C_{i} \backslash S_{k}} a_{C_{i}}\left(x_{C_{i}}\right)
$$

where the summation is over the state-space of variables that are in $C_{i}$ but not in $S_{k}$, and

$$
a_{C_{j}}^{*}\left(x_{C_{j}}\right)=a_{C_{j}}\left(x_{C_{j}}\right) \lambda_{S_{k}}\left(x_{S_{k}}\right)
$$

where

$$
\lambda_{S_{k}}\left(x_{S_{k}}\right)=\frac{b_{S_{k}}^{*}\left(x_{S_{k}}\right)}{b_{S_{k}}\left(x_{S_{k}}\right)} .
$$

$\lambda_{S_{k}}\left(x_{S_{k}}\right)$ is the update factor. Passage of a flow corresponds to updating the neighboring clique with the probability information contained in the originating clique. This flow induces a new representation $K^{*}=\left(\left\{a_{C}^{*}: C \in\right.\right.$ $\left.\left.V_{C}\right\},\left\{b_{S}^{*}: S \in S_{C}\right\}\right)$ for $p(\mathbf{U})$.

A schedule of such flows can be defined such that all cliques are eventually updated with all relevant information and the junction tree reaches an equilibrium state. The most direct scheduling scheme is a two-phase operation where one node is denoted the root of the junction tree. The collection phase involves passing flows along all edges toward the root clique (if a node is scheduled to have more than one incoming flow, the flows are absorbed sequentially). Once collection is complete, the distribution phase involves passing flows out from this root in the reverse direction along the same edges. There are at most two flows along any edge in the tree in a nonredundant schedule. Note that the directionality of the flows in the junction tree need have nothing to do with any directed edges in the original DPIN structure.

5.4 The JLO Algorithm for Inference Given Observed Evidence. The particular case of calculating the effect of observed evidence (inference) is handled in the following manner: Consider that we observe evidence of the form $e=\left\{X_{i}=x_{i}^{*}, X_{j}=x_{j}^{*}, \ldots\right\}$, and $\mathbf{U}^{e}=\left\{X_{i}, X_{j}, \ldots\right\}$ denotes the set of variables observed. Let $\mathbf{U}^{h}=\mathbf{U} \backslash \mathbf{U}^{e}$ denote the set of hidden or unobserved variables and $\mathbf{u}^{h}$ a value assignment for $\mathbf{U}^{h}$.

Consider the calculation of $p\left(\mathbf{U}^{h} \mid e\right)$. Define an evidence function $g^{e}\left(x_{i}\right)$ such that

$$
g^{e}\left(x_{i}\right)= \begin{cases}1 & \text { if } x_{i}=x_{i}^{*} \\ 0 & \text { otherwise }\end{cases}
$$


Let

$$
f^{*}(\mathbf{u})=p(\mathbf{u}) \prod_{\mathbf{U}^{e}} g^{e}\left(x_{i}\right)
$$

Thus, we have that $f^{*}(\mathbf{u}) \propto p\left(\mathbf{u}^{h} \mid e\right)$. To obtain $f^{*}(\mathbf{u})$ by operations on the junction tree, one proceeds as follows: First assign each observed variable $X_{i} \in \mathbf{U}^{e}$ to one particular clique that contains it (this is termed "entering the evidence into the clique"). Let $C^{E}$ denote the set of all cliques into which evidence is entered in this manner. For each $C \in C^{E}$ let

$$
g_{C}\left(x_{C}\right)=\prod_{\left\{i: X_{i} \text { is entered into } C\right\}} g^{e}\left(x_{i}\right)
$$

Thus,

$$
f^{*}(\mathbf{u})=p(\mathbf{u}) \times \prod_{C \in C^{E}} g_{C}\left(x_{C}\right) .
$$

One can now propagate the effects of these modifications throughout the tree using the collect-and-distribute schedule described in Section 5.3. Let $x_{C}^{h}$ denote a value assignment of the hidden (unobserved) variables in clique $C$. When the schedule of flows is complete, one gets a new representation $K_{f}^{*}$ such that the local potential on each clique is $f^{*}\left(x_{C}\right)=p\left(x_{C}^{h}, e\right)$, that is, the joint probability of the local unobserved clique variables and the observed evidence (Jensen et al. 1990) (similarly for the separator potential functions). If one marginalizes at the clique over the unobserved local clique variables,

$$
\sum_{X_{C}^{h}} p\left(x_{C}^{h}, e\right)=p(e)
$$

one gets the probability of the observed evidence directly. Similarly, if one normalizes the potential function at a clique to sum to one, one obtains the conditional probability of the local unobserved clique variables given the evidence, $p\left(x_{C}^{h} \mid e\right)$.

5.5 Complexity of the Propagation Step of the JLO Algorithm. In general, the time complexity $T$ of propagation within a junction tree is $O\left(\sum_{i=1}^{N_{C}} s\left(C_{i}\right)\right)$ where $N_{C}$ is the number of cliques in the junction tree and $s\left(C_{i}\right)$ is the number of states in the clique state-space of $C_{i}$. Thus, for inference to be efficient, we need to construct junction trees with small clique sizes. Problems of finding optimally small junction trees (e.g., finding the junction tree with the smallest maximal clique) are NP-hard. Nonetheless, the heuristic algorithm for triangulation described earlier has been found to work well in practice (Jensen et al. 1990). 


\section{Inference and MAP Calculations in $\operatorname{HMM}(1,1)$}

6.1 The F-B Algorithm for HMM(1,1) Is a Special Case of the JLO Algorithm. Figure $5 \mathrm{~b}$ shows the junction tree for $\operatorname{HMM}(1,1)$. One can apply the JLO algorithm to the $\operatorname{HMM}(1,1)$ junction tree structure to obtain a particular inference algorithm for $\operatorname{HMM}(1,1)$. The $\operatorname{HMM}(1,1)$ inference problem consists of being given a set of values for the observable variables,

$$
e=\left\{O_{1}=o_{1}, O_{2}=o_{2}, \ldots, O_{N}=o_{N}\right\}
$$

and inferring the likelihood of $e$ given the model. As described in the previous section, this problem can be solved exactly by local propagation in any junction tree using the JLO inference algorithm. In Appendix A it is shown that both the forward and backward steps of the F-B procedure for $\operatorname{HMM}(1,1)$ are exactly recreated by the more general JLO algorithm when the $\operatorname{HMM}(1,1)$ is viewed as a PIN.

This equivalence is not surprising since both algorithms are solving exactly the same problem by local recursive updating. The equivalence is useful because it provides a link between well-known HMM inference algorithms and more general PIN inference algorithms. Furthermore, it clearly demonstrates how the PIN framework can provide a direct avenue for analyzing and using more complex hidden Markov probability models (we will discuss such HMMs in Section 8).

When evidence is entered into the observable states and assuming $m$ discrete states per hidden variable, the computational complexity of solving the inference problem via the JLO algorithm is $\mathrm{O}\left(\mathrm{Nm}^{2}\right)$ (the same complexity as the standard F-B procedure).

Note that the obvious structural equivalence between PIN structures and $\operatorname{HMM}(1,1)$ has been noted before by Buntine (1994), Frasconi and Bengio (1994), and Lucke (1995) among others; however, this is the first publication of equivalence of specific inference algorithms as far as we are aware.

6.2 Equivalence of Dawid's Propagation Algorithm for Identifying MAP Assignments and the Viterbi Algorithm. Consider that one wishes to calculate $\hat{f}\left(\mathbf{u}^{h}, e\right)=\max _{x_{1}, \ldots, x_{K}} p\left(x_{1}, \ldots, x_{K}, e\right)$ and also to identify a set of values of the unobserved variables that achieve this maximum, where $K$ is the number of unobserved (hidden) variables. This calculation can be achieved using a local propagation algorithm on the junction tree with two modifications to the standard JLO inference algorithm. This algorithm is due to Dawid (1992a); this is the most general algorithm from a set of related methods.

First, during a flow, the marginalization of the separator is replaced by

$$
\hat{b}_{S}\left(x_{S}\right)=\max _{C \backslash S} a_{C}\left(x_{C}\right)
$$


where $C$ is the originating clique for the flow. The definition for $\lambda_{S}\left(x_{S}\right)$ is also changed in the obvious manner.

Second, marginalization within a clique is replaced by maximization:

$$
\hat{f}_{C}=\max _{\mathbf{u} \backslash x_{C}} p(\mathbf{u}) .
$$

Given these two changes, it can be shown that if the same propagation operations are carried out as described earlier, the resulting representation $\hat{K}_{f}$ at equilibrium is such that the potential function on each clique $C$ is

$$
\hat{f}\left(x_{C}\right)=\max _{\mathbf{u}^{h} \backslash x_{C}} p\left(x_{C}^{h}, e,\left\{\mathbf{u}^{h} \backslash x_{C}\right\}\right),
$$

where $x_{C}^{h}$ denotes a value assignment of the hidden (unobserved) variables in clique $C$. Thus, once the $\hat{K}_{f}$ representation is obtained, one can locally identify the values of $X_{C}^{h}$, which maximize the full joint probability as

$$
\hat{x}_{C}^{h}=\arg _{x_{C}^{h}} \hat{f}\left(x_{C}\right) .
$$

In the probabilistic expert systems literature, this procedure is known as generating the most probable explanation (MPE) given the observed evidence (Pearl 1988).

The $\operatorname{HMM}(1,1)$ MAP problem consists of being given a set of values for the observable variables, $e=\left\{O_{1}=o_{1}, O_{2}=o_{2}, \ldots, O_{N}=o_{N}\right\}$, and inferring

$$
\max _{h_{1}, \ldots, h_{N}} p\left(h_{1}, \ldots, h_{N}, e\right)
$$

or the set of arguments that achieve this maximum. Since Dawid's algorithm is applicable to any junction tree, it can be directly applied to the $\operatorname{HMM}(1,1)$ junction tree in Figure 5b. In Appendix B it is shown that Dawid's algorithm, when applied to $\operatorname{HMM}(1,1)$, is exactly equivalent to the standard Viterbi algorithm. Once again the equivalence is not surprising: Dawid's method and the Viterbi algorithm are both direct applications of dynamic programming to the MAP problem. However, once again, the important point is that Dawid's algorithm is specified for the general case of arbitrary PIN structures and can thus be directly applied to more complex HMMs than $\operatorname{HMM}(1,1)$ (such as those discussed later in Section 8).

\section{Inference and MAP Algorithms for UPINs}

In Section 5 we described the JLO algorithm for local inference given a DPIN. For UPINs the procedure is very similar except for two changes to 
the overall algorithm: the moralization step is not necessary, and initialization of the junction tree is less trivial. In Section 5.2 we described how to go from a specification of conditional probabilities in a directed graph to an initial potential function representation on the cliques in the junction tree. To utilize undirected links in the model specification process requires new machinery to perform the initialization step. In particular we wish to compile the model into the standard form of a product of potentials on the cliques of a triangulated graph (cf. equation 3.1):

$$
P(\mathbf{u})=\prod_{C \in V_{C}} a_{C}\left(x_{C}\right)
$$

Once this initialization step has been achieved, the JLO propagation procedure proceeds as before.

Consider the chordless cycle shown in Figure $4 b$. Suppose that we parameterize the probability distribution on this graph by specifying pairwise marginals on the four pairs of neighboring nodes. We wish to convert such a local specification into a globally consistent joint probability distribution, that is, a marginal representation. An algorithm known as iterative proportional fitting (IPF) is available to perform this conversion. Classically, IPF proceeds as follows (Bishop et al. 1973): Suppose for simplicity that all of the random variables are discrete (a gaussian version of IPF is also available, Whittaker 1990) such that the joint distribution can be represented as a table. The table is initialized with equal values in all of the cells. For each marginal in turn, the table is then rescaled by multiplying every cell by the ratio of the desired marginal to the corresponding marginal in the current table. The algorithm visits each marginal in turn, iterating over the set of marginals. If the set of marginals is consistent with a single joint distribution, the algorithm is guaranteed to converge to the joint distribution. Once the joint is available, the potentials in equation 3.1 can be obtained (in principle) by marginalization.

Although IPF solves the initialization problem in principle, it is inefficient. Jiřousek and Přeučil (1995) developed an efficient version of IPF that avoids the need for both storing the joint distribution as a table and explicit marginalization of the joint to obtain the clique potentials. Jiřousek's version of IPF represents the evolving joint distribution directly in terms of junction tree potentials. The algorithm proceeds as follows: Let $\mathcal{I}$ be a set of subsets of $V$. For each $I \in \mathcal{I}$, let $q\left(x_{I}\right)$ denote the desired marginal on the subset $I$. Let the joint distribution be represented as a product over junction tree potentials (see equation 3.1), where each $a_{C}$ is initialized to an arbitrary constant. Visit each $I \in \mathcal{I}$ in turn, updating the corresponding clique potential $a_{C}$ (i.e, that potential $a_{C}$ for which $I \subseteq C$ ) as follows:

$$
a_{C}^{*}\left(x_{C}\right)=a_{C}\left(x_{C}\right) \frac{q\left(x_{I}\right)}{p\left(x_{I}\right)} .
$$


The marginal $p\left(x_{I}\right)$ is obtained via the JLO algorithm, using the current set of clique potentials. Intelligent choices can be made for the order in which to visit the marginals to minimize the amount of propagation needed to compute $p\left(x_{I}\right)$. This algorithm is simply an efficient way of organizing the IPF calculations and inherits the latter's guarantees of convergence.

Note that the Jiřousek and Přeučil algorithm requires a triangulation step in order to form the junction tree used in the calculation of $p\left(x_{I}\right)$. In the worst case, triangulation can yield a highly connected graph, in which case the Jiřousek and Přeučil algorithm reduces to classical IPF. For sparse graphs, however, when the maximum clique is much smaller than the entire graph, the algorithm should be substantially more efficient than classical IPF. Moreover, the triangulation algorithm itself need only be run once as a preprocessing step (as is the case for the JLO algorithm).

\section{More Complex HMMs for Speech Modeling}

Although HMMs have provided an exceedingly useful framework for the modeling of speech signals, it is also true that the simple $\operatorname{HMM}(1,1)$ model underlying the standard framework has strong limitations as a model of speech. Real speech is generated by a set of coupled dynamical systems (lips, tongue, glottis, lungs, air columns, etc.), each obeying particular dynamical laws. This coupled physical process is not well modeled by the unstructured state transition matrix of $\operatorname{HMM}(1,1)$. Moreover, the first-order Markov properties of $\operatorname{HMM}(1,1)$ are not well suited to modeling the ubiquitous coarticulation effects that occur in speech, particularly coarticulatory effects that extend across several phonemes (Kent and Minifie 1977). A variety of techniques have been developed to surmount these basic weaknesses of the $\operatorname{HMM}(1,1)$ model, including mixture modeling of emission probabilities, triphone modeling, and discriminative training. All of these methods, however, leave intact the basic probabilistic structure of $\operatorname{HMM}(1,1)$ as expressed by its PIN structure.

In this section we describe several extensions of $\operatorname{HMM}(1,1)$ that assume additional probabilistic structure beyond that assumed by $\operatorname{HMM}(1,1)$. PINs provide a key tool in the study of these more complex models. The role of PINs is twofold: they provide a concise description of the probabilistic dependencies assumed by a particular model, and they provide a general algorithm for computing likelihoods. This second property is particularly important because the existence of the JLO algorithm frees us from having to derive particular recursive algorithms on a case-by-case basis.

The first model that we consider can be viewed as a coupling of two $\operatorname{HMM}(1,1)$ chains (Saul and Jordan, 1995). Such a model can be useful in general sensor fusion problems, for example, in the fusion of an audio signal with a video signal in lipreading. Because different sensory signals generally have different bandwidths, it may be useful to couple separate Markov models that are developed specifically for each of the individual signals. The 
alternative is to force the problem into an $\operatorname{HMM}(1,1)$ framework by either oversampling the slower signal, which requires additional parameters and leads to a high-variance estimator, or downsampling the faster signal, which generally oversmoothes the data and yields a biased estimator. Consider the $\operatorname{HMM}(1,2)$ structure shown in Figure 8a. This model involves two $\operatorname{HMM}(1,1)$ backbones that are coupled together by undirected links between the state variables. Let $H_{i}^{(1)}$ and $O_{i}^{(1)}$ denote the $i$ th state and $i$ th output of the "fast" chain, respectively, and let $H_{i}^{(2)}$ and $O_{i}^{(2)}$ denote the $i$ th state and $i$ th output of the "slow" chain. Suppose that the fast chain is sampled $\tau$ times as often as the slow chain. Then $H_{i^{\prime}}^{(1)}$ is connected to $H_{i}^{(2)}$ for $i^{\prime}$ equal to $\tau(i-1)+1$. Given this value for $i^{\prime}$, the Markov model for the coupled chain implies the following conditional independencies for the state variables:

$$
\begin{gathered}
\left\{H_{i^{\prime}}^{(1)}, H_{i}^{(2)}\right\} \perp\left\{H_{1}^{(1)}, O_{1}^{(1)}, H_{1}^{(2)}, O_{1}^{(2)}, \ldots, H_{i^{\prime}-2}^{(1)}, O_{i^{\prime}-2}^{(1)}, H_{i-2}^{(2)}, O_{i-2}^{(2)},\right. \\
\left.O_{i^{\prime}-1}^{(1)}, O_{i-1}^{(2)}\right\} \mid\left\{H_{i^{\prime}-1}^{(1)}, H_{i-1}^{(2)}\right\},
\end{gathered}
$$

as well as the following conditional independencies for the output variables:

$$
\begin{gathered}
\left\{O_{i^{\prime}}^{(1)}, O_{i}^{(2)}\right\} \perp\left\{H_{1}^{(1)}, O_{1}^{(1)}, H_{1}^{(2)}, O_{1}^{(2)}, \ldots, H_{i^{\prime}-1}^{(1)}, O_{i^{\prime}-1}^{(1)},\right. \\
\left.H_{i-1}^{(2)}, O_{i-1}^{(2)}\right\} \mid\left\{H_{i^{\prime}}^{(1)}, H_{i}^{(2)}\right\} .
\end{gathered}
$$

Additional conditional independencies can be read off the UPIN structure (see Figure 8a).

As is readily seen in Figure $8 \mathrm{a}$, the $\operatorname{HMM}(1,2)$ graph is not triangulated; thus, the $\operatorname{HMM}(1,2)$ probability model is not decomposable. However, the graph can be readily triangulated to form a decomposable cover for the $\operatorname{HMM}(1,2)$ probability model (see Section 3.1.2). The JLO algorithm provides an efficient algorithm for calculating likelihoods in this graph. This can be seen in Figure 8b, where we show a triangulation of the $\operatorname{HMM}(1,2)$ graph. The triangulation adds $O\left(N_{h}\right)$ links to the graph (where $N_{h}$ is the number of hidden nodes in the graph) and creates a junction tree in which each clique is a cluster of three state variables from the underlying UPIN structure. Assuming $m$ values for each state variable in each chain, we obtain an algorithm whose time complexity is $O\left(N_{h} m^{3}\right)$. This can be compared to the naive approach of transforming the $\operatorname{HMM}(1,2)$ model to a Cartesian product $\operatorname{HMM}(1,1)$ model, which not only has the disadvantage of requiring subsampling or oversampling but also has a time complexity of $O\left(N_{h} m^{4}\right)$.

Directed graph semantics can also play an important role in constructing interesting variations on the HMM theme. Consider Figure 9a, which shows an $\operatorname{HMM}(1,2)$ model in which a single output stream is coupled to a pair of underlying state sequences. In a speech modeling application, such a structure might be used to capture the fact that a given acoustic pattern can have multiple underlying articulatory causes. For example, equivalent shifts in 
(a)

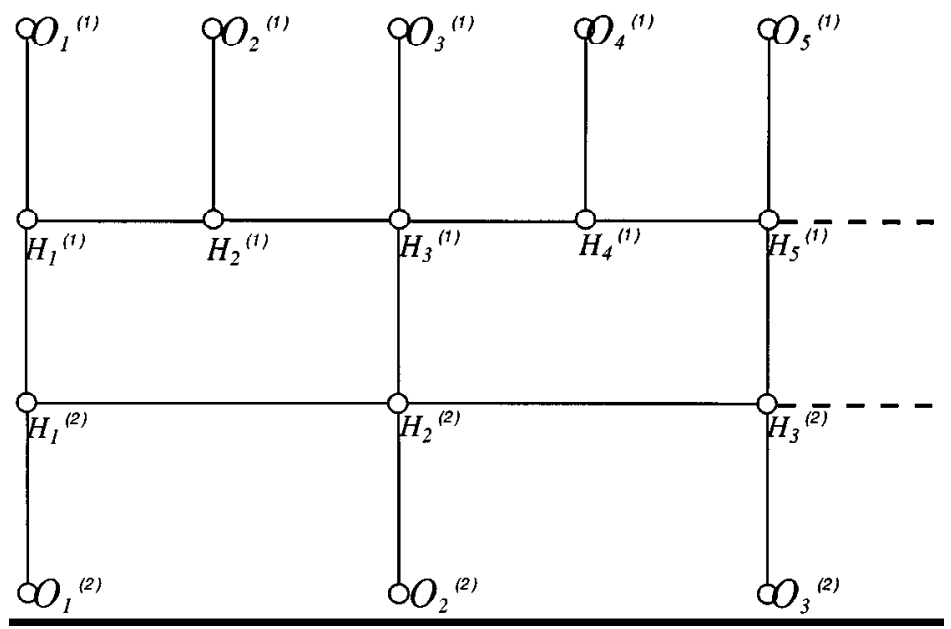

(b)

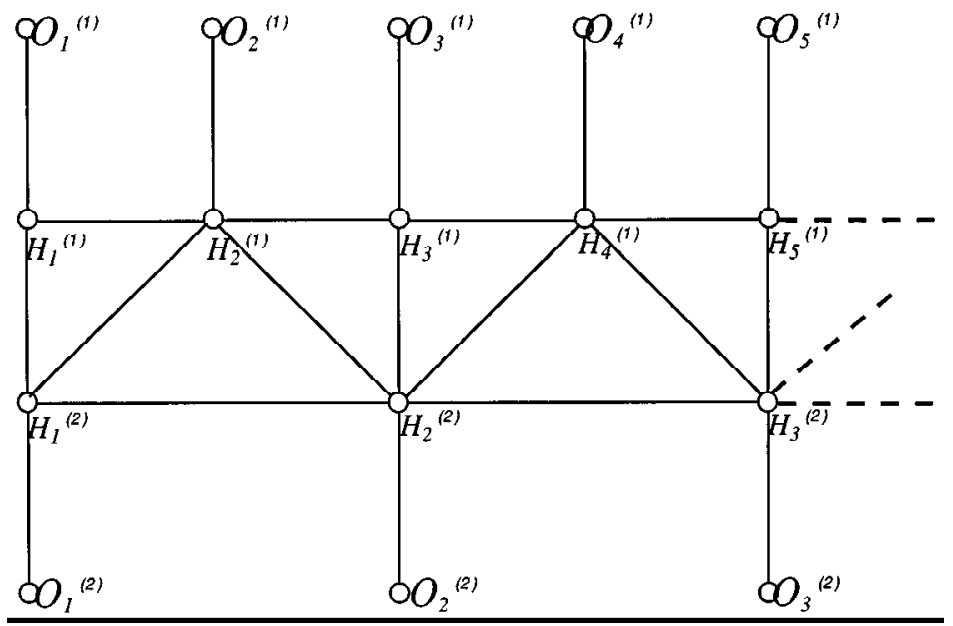

Figure 8: (a) The UPIN structure for the $\operatorname{HMM}(1,2)$ model with $\tau=2$. (b) A triangulation of this UPIN structure. 
formant frequencies can be caused by lip rounding or tongue raising; such phenomena are generically refered to as "trading relations" in the speech psychophysics literature (Lindblom 1990; Perkell et al. 1993). Once a particular acoustic pattern is observed, the causes become dependent; thus, for example, evidence that the lips are rounded would act to discount inferences that the tongue has been raised. These inferences propagate forward and backward in time and couple the chains. Formally, these induced dependencies are accounted for by the links added between the state sequences during the moralization of the graph (see Figure $9 b$ ). This figure shows that the underlying calculations for this model are closely related to those of the earlier $\operatorname{HMM}(1,2)$, but the model specification is very different in the two cases.

Saul and Jordan (1996) have proposed a second extension of the $\operatorname{HMM}(1,1)$ model that is motivated by the desire to provide a more effective model of coarticulation (see also Stolorz 1994). In this model, shown in Figure 10, coarticulatory influences are modeled by additional links between output variables and states along an $\operatorname{HMM}(1,1)$ backbone. One approach to performing calculations in this model is to treat it as a Kth-order Markov chain and transform it into an $\operatorname{HMM}(1,1)$ model by defining higher-order state variables. A graphical modeling approach is more flexible. It is possible, for example, to introduce links between states and outputs $K$ time steps apart without introducing links for the intervening time intervals. More generally, the graphical modeling approach to the $\operatorname{HMM}(K, 1)$ model allows the specification of different interaction matrices at different time scales; this is awkward in the Kth-order Markov chain formalism.

The $\operatorname{HMM}(3,1)$ graph is triangulated as is, and thus the time complexity of the JLO algorithm is $O\left(N_{h} m^{3}\right)$. In general an $\operatorname{HMM}(K, 1)$ graph creates cliques of size $O\left(m^{K}\right)$, and the JLO algorithm runs in time $O\left(N_{h} m^{K}\right)$.

As these examples suggest, the graphical modeling framework provides a useful framework for exploring extensions of HMMs. The examples also make clear, however, that the graphical algorithms are no panacea. The $m^{K}$ complexity of $\operatorname{HMM}(\mathrm{K}, 1)$ will be prohibitive for large $K$. Also, the generalization of $\operatorname{HMM}(1,2)$ to $\operatorname{HMM}(1, \mathrm{~K})$ (couplings of $K$ chains) is intractable. Recent research has therefore focused on approximate algorithms for inference in such structures; see Saul and Jordan (1996) for $\operatorname{HMM}(\mathrm{K}, 1)$ and Ghahramani and Jordan (1996) and Williams and Hinton (1990) for $\operatorname{HMM}(1, K)$. These authors have developed an approximation methodology based on mean-field theory from statistical physics. While discussion of mean-field algorithms is beyond the scope of this article, it is worth noting that the graphical modeling framework plays a useful role in the development of these approximations. Essentially the mean-field approach involves creating a simplified graph for which tractable algorithms are available, and minimizing a probabilistic distance between the tractable graph and the intractable graph. The JLO algorithm is called as a subroutine on the tractable graph during the minimization process. 


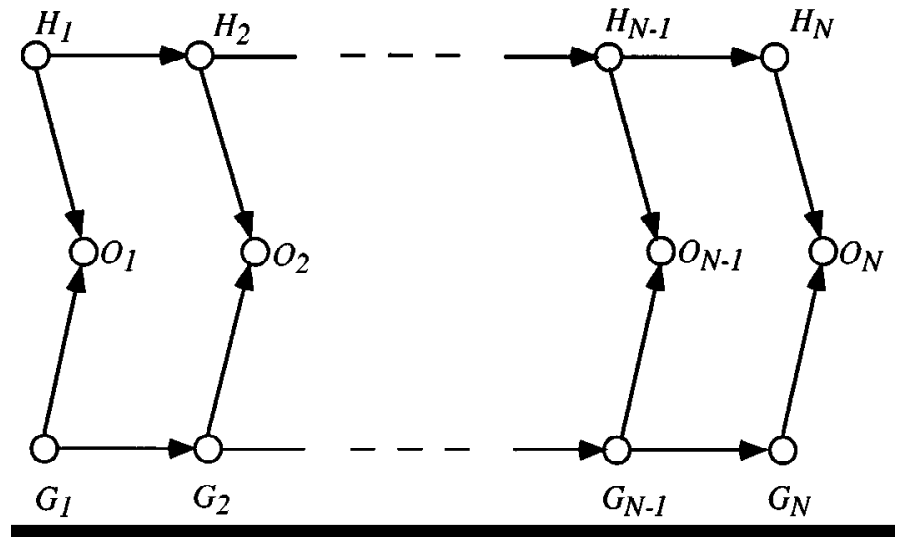

(a)

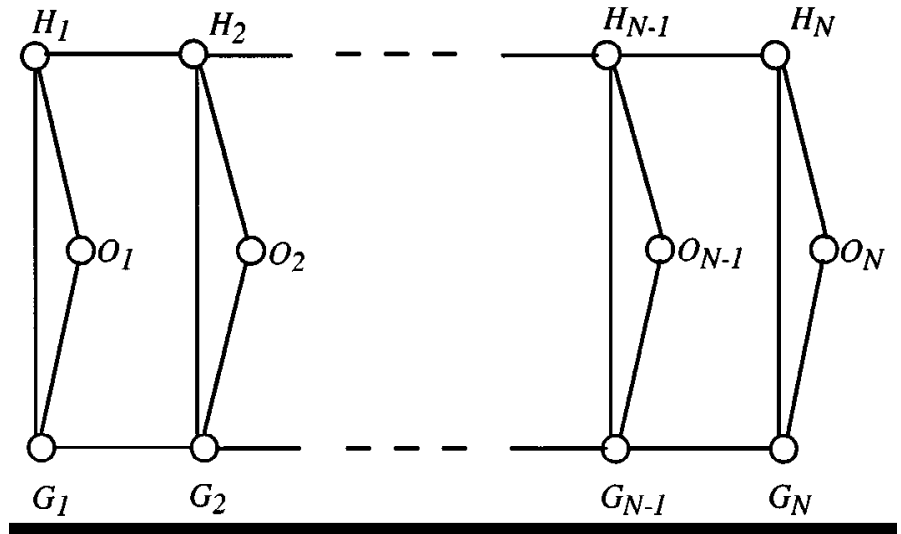

(b)

Figure 9: (a) The DPIN structure for $\operatorname{HMM}(1,2)$ with a single observable sequence coupled to a pair of underlying state sequences. (b) The moralization of this DPIN structure.

\section{Learning and PINs}

Until now, we have assumed that the parameters and structure of a PIN are known with certainty. In this section, we drop this assumption and discuss methods for learning about the parameters and structure of a PIN.

The basic idea behind the techniques that we discuss is that there is a true joint probability distribution described by some PIN structure and parameters, but we are uncertain about this structure and its parameters. We 


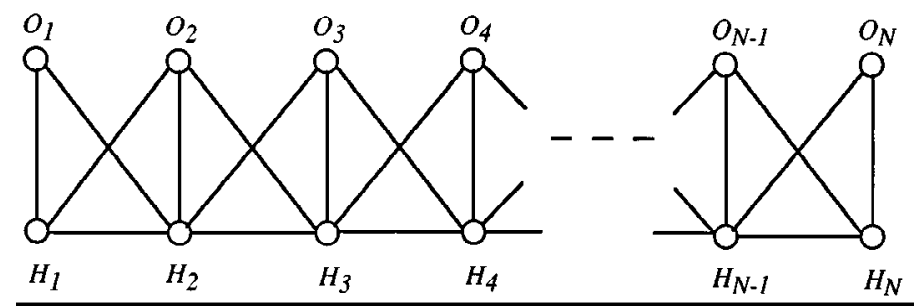

Figure 10: The UPIN structure for $\operatorname{HMM}(3,1)$.

are unable to observe the true joint distribution directly, but we are able to observe a set of patterns $\mathbf{u}_{1}, \ldots, \mathbf{u}_{M}$ that is a random sample from this true distribution. These patterns are independent and identically distributed (i.i.d.) according to the true distribution (note that in a typical HMM learning problem, each of the $\mathbf{u}_{i}$ consist of a sequence of observed data). We use these data to learn about the structure and parameters that encode the true distribution.

9.1 Parameter Estimation for PINs. First, let us consider the situation where we know the PIN structure $S$ of the true distribution with certainty but are uncertain about the parameters of $S$.

In keeping with the rest of the article, let us assume that all variables in $\mathbf{U}$ are discrete. Furthermore, for purposes of illustration, let us assume that $S$ is an ADG. Let $x_{i}^{k}$ and $p a\left(X_{i}\right)^{j}$ denote the $k$ th value of variable $X_{i}$ and $j$ th configuration of variables $p a\left(X_{i}\right)$ in $S$, respectively $\left(j=1, \ldots, q_{i}\right.$, $\left.k=1, \ldots, r_{i}\right)$. As we have just discussed, we assume that each conditional probability $p\left(x_{i}^{k} \mid p a\left(X_{i}\right)^{j}\right)$ is an uncertain parameter, and for convenience we represent this parameter as $\theta_{i j k}$. We use $\boldsymbol{\theta}_{i j}$ to denote the vector of parameters $\left(\theta_{i j 1}, \ldots, \theta_{i j r_{i}}\right)$ and $\boldsymbol{\theta}_{s}$ to denote the vector of all parameters for $S$. Note that $\sum_{k=1}^{r_{i}} \theta_{i j k}=1$ for every $i$ and $j$.

One method for learning about the parameters $\boldsymbol{\theta}_{s}$ is the Bayesian approach. We treat the parameters $\boldsymbol{\theta}_{s}$ as random variables, assign these parameters a prior distribution $p\left(\boldsymbol{\theta}_{S} \mid S\right)$, and update this prior distribution with data $D=\left(\mathbf{u}_{1}, \ldots, \mathbf{u}_{M}\right)$ according to Bayes' rule:

$$
p\left(\boldsymbol{\theta}_{S} \mid D, S\right)=c \cdot p\left(\boldsymbol{\theta}_{s} \mid S\right) p\left(D \mid \boldsymbol{\theta}_{s}, S\right),
$$

where $c$ is a normalization constant that does not depend on $\boldsymbol{\theta}_{s}$. Because the patterns in $D$ are a random sample, equation 9.1 simplifies to

$$
p\left(\boldsymbol{\theta}_{S} \mid D, S\right)=c \cdot p\left(\boldsymbol{\theta}_{S} \mid S\right) \prod_{l=1}^{M} p\left(\mathbf{u}_{l} \mid \boldsymbol{\theta}_{S}, S\right) .
$$




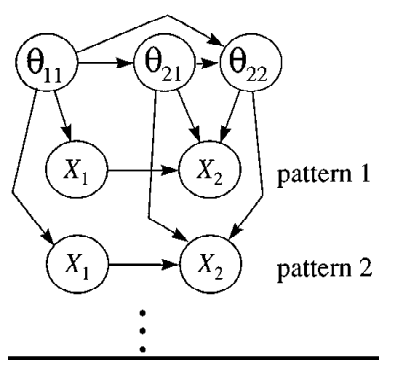

(a)

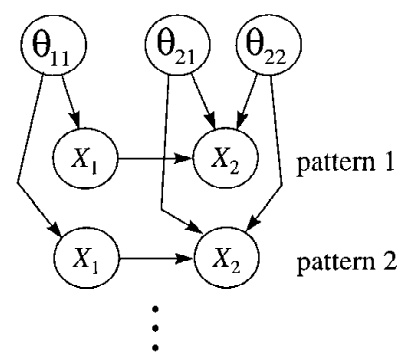

(b)

Figure 11: A Bayesian network structure for a two-binary-variable domain $\left\{X_{1}, X_{2}\right\}$ showing (a) conditional independencies associated with the random sample assumption and (b) the added assumption of parameter independence. In both parts of the figure, it is assumed that the network structure $X_{1} \rightarrow X_{2}$ is generating the patterns.

Given some prediction of interest that depends on $\boldsymbol{\theta}_{S}$ and $S$ - say, $f\left(\boldsymbol{\theta}_{S}, S\right)$ we can use the posterior distribution of $\boldsymbol{\theta}_{s}$ to compute an expected prediction:

$$
E\left(f\left(\boldsymbol{\theta}_{s}, S\right) \mid D, S\right)=\int f\left(\boldsymbol{\theta}_{s}, S\right) p\left(\boldsymbol{\theta}_{s} \mid D, S\right) d \boldsymbol{\theta}_{s} .
$$

Associated with our assumption that the data $D$ are a random sample from structure $S$ with uncertain parameters $\boldsymbol{\theta}_{S}$ is a set of conditional independence assertions. Not surprisingly, some of these assumptions can be represented as a (directed) PIN that includes both the possible observations and the parameters as variables. Figure 11a shows these assumptions for the case where $\mathbf{U}=\left\{X_{1}, X_{2}\right\}$ and $S$ is the structure with a directed edge from $X_{1}$ to $X_{2}$.

Under certain additional assumptions, described, for example, in Spiegelhalter and Lauritzen (1990), the evaluation of equation 9.2 is straightfor- 
ward. In particular, if each pattern $\mathbf{u}_{l}$ is complete (i.e., every variable is observed), we have

$$
p\left(\mathbf{u}_{l} \mid \boldsymbol{\theta}_{s}, S\right)=\prod_{i=1}^{N} \prod_{j=1}^{q_{i}} \prod_{k=1}^{r_{i}} \theta_{i j k}^{\delta_{i j k l}}
$$

where $\delta_{i j k l}$ is equal to one if $X_{i}=x_{i}^{k}$ and $p a\left(X_{i}\right)=p a\left(X_{i}\right)^{j}$ in pattern $C_{l}$ and zero otherwise. Combining equations 9.2 and 9.4 , we obtain

$$
p\left(\boldsymbol{\theta}_{s} \mid D, S\right)=c \cdot p\left(\boldsymbol{\theta}_{S} \mid S\right) \prod_{i=1}^{N} \prod_{j=1}^{q_{i}} \prod_{k=1}^{r_{i}} \theta_{i j k}^{N_{i j k}},
$$

where $N_{i j k}$ is the number of patterns in which $X_{i}=x_{i}^{k}$ and $p a\left(X_{i}\right)=p a\left(X_{i}\right)^{j}$. The $N_{i j k}$ are the sufficient statistics for the random sample $D$. If we assume that the parameter vectors $\boldsymbol{\theta}_{i j}, i=1, \ldots, n, j=1, \ldots, q_{i}$ are mutually independent, an assumption we call parameter independence, then we get the additional simplification

$$
p\left(\boldsymbol{\theta}_{S} \mid D, S\right)=c \prod_{i=1}^{N} \prod_{j=1}^{q_{i}} p\left(\boldsymbol{\theta}_{i j} \mid S\right) \prod_{k=1}^{r_{i}} \theta_{i j k}^{N_{i j k}}
$$

The assumption of parameter independence for our two-variable example is illustrated in Figure 11b. Thus, given complete data and parameter independence, each parameter vector $\boldsymbol{\theta}_{i j}$ can be updated independently. The update is particularly simple if each parameter vector has a conjugate distribution. For a discrete variable with discrete parents, the natural conjugate distribution is the Dirichlet,

$$
p\left(\boldsymbol{\theta}_{i j} \mid S\right) \propto \prod_{k=1}^{r_{i}} \theta_{i j k}^{\alpha_{i j k}-1},
$$

in which case equation 9.6 becomes

$$
p\left(\boldsymbol{\theta}_{S} \mid D, S\right)=c \prod_{i=1}^{N} \prod_{j=1}^{q_{i}} \prod_{k=1}^{r_{i}} \theta_{i j k}^{N_{i j k}+\alpha_{i j k}-1} .
$$

Other conjugate distributions include the normal Wishart distribution for the parameters of gaussian codebooks and the Dirichlet distribution for the mixing coefficients of gaussian-mixture codebooks (DeGroot 1970; Buntine 1994; Heckerman and Geiger 1995). Heckerman and Geiger (1995) describe a simple method for assessing these priors. These priors have also been used for learning parameters in standard HMMs (e.g., Gauvain and Lee 1994). 
Parameter independence is usually not assumed in general for HMM structures. For example, in the $\operatorname{HMM}(1,1)$ model, a standard assumption is that $p\left(H_{i} \mid H_{i-1}\right)=p\left(H_{j} \mid H_{j-1}\right)$ and $p\left(O_{i} \mid H_{i}\right)=p\left(O_{j} \mid H_{j}\right)$ for all appropriate $i$ and $j$. Fortunately, parameter equalities such as these are easily handled in the framework above (see Thiesson 1995 for a detailed discussion).

In addition, the assumption that patterns are complete is clearly inappropriate for HMM structures in general, where some of the variables are hidden from observation. When data are missing, the exact evaluation of the posterior $p\left(\boldsymbol{\theta}_{S} \mid D, S\right)$ is typically intractable, so we turn to approximations. Accurate but slow approximations are based on Monte Carlo sampling (e.g., Neal 1993). An approximation that is less accurate but more efficient is one based on the observation that, under certain conditions, the quantity $p\left(\boldsymbol{\theta}_{S} \mid S\right) \cdot p\left(D \mid \boldsymbol{\theta}_{S}, S\right)$ converges to a multivariate gaussian distribution as the sample size increases (see, e.g., Kass et al. 1988; MacKay, 1992a, 1992b).

Less accurate but more efficient approximations are based on the observation that the gaussian distribution converges to a delta function centered at the maximum a posteriori (MAP) and eventually the maximum likelihood (ML) value of $\boldsymbol{\theta}_{s}$. For the standard $\operatorname{HMM}(1,1)$ model discussed in this article, where either discrete, gaussian, or gaussian-mixture codebooks are used, an ML or MAP estimate is a well-known efficient approximation (Poritz 1988; Rabiner 1989).

MAP and ML estimates can be found using traditional techniques such as gradient descent and expectation-maximization (EM) (Dempster et al., 1977). The EM algorithm can be applied efficiently whenever the likelihood function has sufficient statistics that are of fixed dimension for any data set. The EM algorithm finds a local maximum by initializing the parameters $\boldsymbol{\theta}_{s}$ (e.g., at random or by some clustering algorithm) and repeating $\mathrm{E}$ and $\mathrm{M}$ steps to convergence. In the E step, we compute the expected sufficient statistic for each of the parameters, given $D$ and the current values for $\boldsymbol{\theta}_{s}$. In particular, if all variables are discrete and parameter independence is assumed to hold, and all priors are Dirichlet, we obtain

$$
E\left(N_{i j k} \mid D, \boldsymbol{\theta}_{s}, S\right)=\sum_{l=1}^{M} p\left(x_{i}^{k}, p a\left(X_{i}\right)^{j} \mid \mathbf{u}_{l}, \boldsymbol{\theta}_{s}, S\right)
$$

An important feature of the EM algorithm applied to PINs under these assumptions is that each term in the sum can be computed using the JLO algorithm. The JLO algorithm may also be used when some parameters are equal and when the likelihoods of some variables are gaussian or gaussianmixture distributions (Lauritzen and Wermuth 1989). In the M step, we use the expected sufficient statistics as if they were actual sufficient statistics and set the new values of $\boldsymbol{\theta}_{s}$ to be the MAP or ML values given these statistics. Again, if all variables are discrete, parameter independence is assumed to 
hold, and all priors are Dirichlet, the ML is given by

$$
\theta_{i j k}=\frac{E\left(N_{i j k} \mid D, \boldsymbol{\theta}_{s}, S\right)}{\sum_{k=1}^{r_{i}} E\left(N_{i j k} \mid D, \boldsymbol{\theta}_{s}, S\right)},
$$

and the MAP is given by

$$
\theta_{i j k}=\frac{E\left(N_{i j k} \mid D, \boldsymbol{\theta}_{s}, S\right)+\alpha_{i j k}-1}{\sum_{k=1}^{r_{i}}\left(E\left(N_{i j k} \mid D, \boldsymbol{\theta}_{s}, S\right)+\alpha_{i j k}-1\right)} .
$$

9.2 Model Selection and Averaging for PINs. Now let us assume that we are uncertain not only about the parameters of a PIN but also about the true structure of a PIN. For example, we may know that the true structure is an $\operatorname{HMM}(K, J)$ structure, but we may be uncertain about the values of $K$ and $J$.

One solution to this problem is Bayesian model averaging. In this approach, we view each possible PIN structure (without its parameters) as a model. We assign prior probabilities $p(S)$ to different models, and compute their posterior probabilities given data:

$$
p(S \mid D) \propto p(S) p(D \mid S)=p(S) \int p(D \mid \boldsymbol{\theta}, S) p(\boldsymbol{\theta} \mid S) d \boldsymbol{\theta} .
$$

As indicated in equation 9.8, we compute $p(D \mid S)$ by averaging the likelihood of the data over the parameters of $S$. In addition to computing the posterior probabilities of models, we estimate the parameters of each model either by computing the distribution $p(\boldsymbol{\theta} \mid D, S)$ or using a gaussian, MAP, or ML approximation for this distribution. We then make a prediction of interest based on each model separately, as in equation 9.3, and compute the weighted average of these predictions using the posterior probabilities of models as weights.

One complication with this approach is that when data are missing-for example, when some variables are hidden-the exact computation of the integral in equation 9.8 is usually intractable. As discussed in the previous section, Monte Carlo and gaussian approximations may be used. One simple form of a gaussian approximation is the Bayesian information criterion (BIC) described by Schwarz (1978),

$$
\log p(D \mid S) \approx \log p\left(D \mid \hat{\boldsymbol{\theta}}_{s}, S\right)-\frac{d}{2} \log M
$$

where $\hat{\boldsymbol{\theta}}_{S}$ is the ML estimate, $M$ is the number of patterns in $D$, and $d$ is the dimension of $S$-typically, the number of parameters of $S$. The first term of this "score" for $S$ rewards how well the data fit $S$, whereas the second term punishes model complexity. Note that this score does not depend on the 
parameter prior, and thus can be applied easily. ${ }^{3}$ For examples of applications of BIC in the context of PINs and other statistical models, see Raftery (1995).

The BIC score is the additive inverse of Rissanen's (1987) minimum description length (MDL). Other scores, which can be viewed as approximations to the marginal likelihood, are hypothesis testing (Raftery 1995) and cross validation (Dawid 1992b). Buntine (in press) provides a comprehensive review of scores for model selection and model averaging in the context of PINs.

Another complication with Bayesian model averaging is that there may be so many possible models that averaging becomes intractable. In this case, we select one or a handful of structures with high relative posterior probabilities and make our predictions with this limited set of models. This approach is called model selection. The trick here is finding a model or models with high posterior probabilities. Detailed discussions of search methods for model selection among PINs are given by, among others, Madigan and Raftery (1994), Heckerman et al. (1995), and Spirtes and Meek (1995). When the true model is some $\operatorname{HMM}(K, J)$ structure, we may have additional prior knowledge that strongly constrains the possible values of $K$ and $J$. Here, exhaustive model search is likely to be practical.

\section{Summary}

Probabilistic independence networks provide a useful framework for both the analysis and application of multivariate probability models when there is considerable structure in the model in the form of conditional independence. The graphical modeling approach both clarifies the independence semantics of the model and yields efficient computational algorithms for probabilistic inference. This article has shown that it is useful to cast HMM structures in a graphical model framework. In particular, the well-known F-B and Viterbi algorithms were shown to be special cases of more general algorithms from the graphical modeling literature. Furthermore, more complex HMM structures, beyond the traditional first-order model, can be analyzed profitably and directly using generally applicable graphical modeling techniques.

\section{Appendix A: The Forward-Backward Algorithm for $\operatorname{HMM}(1,1)$ Is a Special Case of the JLO Algorithm}

Consider the junction tree for $\operatorname{HMM}(1,1)$ as shown in Figure $5 b$. Let the final clique in the chain containing $\left(H_{N-1}, H_{N}\right)$ be the root clique. Thus, a

\footnotetext{
${ }^{3}$ One caveat: The BIC score is derived under the assumption that the parameter prior is positive throughout its domain.
} 
nonredundant schedule consists of first recursively passing flows from each $\left(\mathrm{O}_{i}, H_{i}\right)$ and $\left(H_{i-2}, H_{i-1}\right)$ to each $\left(H_{i-1}, H_{i}\right)$ in the appropriate sequence (the "collect" phase), and then distributing flows out in the reverse direction from the root clique. If we are interested only in calculating the likelihood of $e$ given the model, then the distribute phase is not necessary since we can simply marginalize over the local variables in the root clique to obtain $p(e)$. (Subscripts on potential functions and update factors indicate which variables have been used in deriving that potential or update factor; e.g., $f_{O_{1}}$ indicates that this potential has been updated based on information about $\mathrm{O}_{1}$ but not using information about any other variables.)

Assume that the junction tree has been initialized so that the potential function for each clique and separator is the local marginal. Given the observed evidence $e$, each individual piece of evidence $O=o_{i}^{*}$ is entered into its clique $\left(O_{i}, H_{i}\right)$ such that each clique marginal becomes $f_{O_{i}}^{*}\left(h_{i}, o_{i}\right)=p\left(h_{i}, o_{i}^{*}\right)$ after entering the evidence (as in equation 5.8).

Consider the portion of the junction tree in Figure 12, and in particular the flow between $\left(O_{i}, H_{i}\right)$ and $\left(H_{i-1}, H_{i}\right)$. By definition the potential on the separator $H_{i}$ is updated to

$$
f_{O_{i}}^{*}\left(h_{i}\right)=\sum_{o_{i}} f^{*}\left(h_{i}, o_{i}\right)=p\left(h_{i}, o_{i}^{*}\right)
$$

The update factor from this separator flowing into clique $\left(H_{i-1}, H_{i}\right)$ is then

$$
\lambda_{O_{i}}\left(h_{i}\right)=\frac{p\left(h_{i}, o_{i}^{*}\right)}{p\left(h_{i}\right)}=p\left(o_{i}^{*} \mid h_{i}\right) .
$$

This update factor is "absorbed" into $\left(H_{i-1}, H_{i}\right)$ as follows:

$$
f_{O_{i}}^{*}\left(h_{i-1}, h_{i}\right)=p\left(h_{i-1}, h_{i}\right) \lambda_{O_{i}}\left(h_{i}\right)=p\left(h_{i-1}, h_{i}\right) p\left(o_{i}^{*} \mid h_{i}\right)
$$

Now consider the flow from clique $\left(H_{i-2}, H_{i-1}\right)$ to clique $\left(H_{i-1}, H_{i}\right)$. Let $\Phi_{i, j}=\left\{O_{i}, \ldots, O_{j}\right\}$ denote a set of consecutive observable variables and $\phi_{i, j}^{*}=\left\{o_{i}^{*}, \ldots, o_{j}^{*}\right\}$ denote a set of observed values for these variables, $1 \leq$ $i<j \leq N$. Assume that the potential on the separator $H_{i-1}$ has been updated to

$$
f_{\Phi_{1, i-1}}^{*}\left(h_{i-1}\right)=p^{*}\left(h_{i-1}, \phi_{1, i-1}^{*}\right)
$$

by earlier flows in the schedule. Thus, the update factor on separator $H_{i-1}$ becomes

$$
\lambda_{\Phi_{1, i-1}}\left(h_{i-1}\right)=\frac{p^{*}\left(h_{i-1}, \phi_{1, i-1}^{*}\right)}{p\left(h_{i-1}\right)},
$$




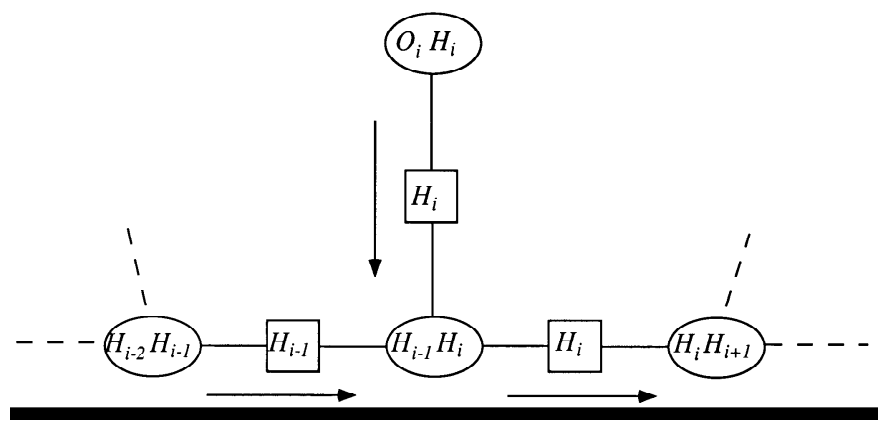

Figure 12: Local message passing in the $\operatorname{HMM}(1,1)$ junction tree during the collect phase of a left-to-right schedule. Ovals indicate cliques, boxes indicate separators, and arrows indicate flows.

and this gets absorbed into clique $\left(H_{i-1}, H_{i}\right)$ to produce

$$
\begin{aligned}
f_{\Phi_{1, i}}^{*}\left(h_{i-1}, h_{i}\right) & =f_{O_{i}}^{*}\left(h_{i-1}, h_{i}\right) \lambda_{\Phi_{1, i-1}}\left(h_{i-1}\right) \\
& =p\left(h_{i-1}, h_{i}\right) p\left(o_{i}^{*} \mid h_{i}\right) \frac{p^{*}\left(h_{i-1}, \phi_{1, i-1}^{*}\right)}{p\left(h_{i-1}\right)} \\
& =p\left(o_{i}^{*} \mid h_{i}\right) p\left(h_{i} \mid h_{i-1}\right) p^{*}\left(h_{i-1}, \phi_{1, i-1}^{*}\right) .
\end{aligned}
$$

Finally, we can calculate the new potential on the separator for the flow from clique $\left(H_{i-1}, H_{i}\right)$ to $\left(H_{i}, H_{i+1}\right)$ :

$$
\begin{aligned}
f_{\Phi_{1, i}}^{*}\left(h_{i}\right) & =\sum_{h_{i-1}} f_{\Phi_{1, i}}^{*}\left(h_{i-1}, h_{i}\right) \\
& =p\left(o_{i}^{*} \mid h_{i}\right) \sum_{h_{i-1}} p\left(h_{i} \mid h_{i-1}\right) p^{*}\left(h_{i-1}, \phi_{1, i-1}^{*}\right) \\
& =p\left(o_{i}^{*} \mid h_{i}\right) \sum_{h_{i-1}} p\left(h_{i} \mid h_{i-1}\right) f_{\Phi_{1, i-1}}^{*}\left(h_{i-1}\right)
\end{aligned}
$$

Proceeding recursively in this manner, one finally obtains at the root clique

$$
f_{\Phi_{1, N}}^{*}\left(h_{N-1}, h_{N}\right)=p\left(h_{N-1}, h_{N}, \phi_{1, N}^{*}\right)
$$

from which one can get the likelihood of the evidence,

$$
p(e)=p\left(\phi_{1, N}^{*}\right)=\sum_{h_{N-1}, h_{N}} f_{\Phi_{1, N}}^{*}\left(h_{N-1}, h_{N}\right) .
$$


We note that equation A.9 directly corresponds to the recursive equation (equation 20 in Rabiner 1989) for the $\alpha$ variables used in the forward phase of the F-B algorithm, the standard $\operatorname{HMM}(1,1)$ inference algorithm. In particular, using a "left-to-right" schedule, the updated potential functions on the separators between the hidden cliques, the $f_{\Phi_{1, i}}^{*}\left(h_{i}\right)$ functions, are exactly the $\alpha$ variables. Thus, when applied to $\operatorname{HMM}(1,1)$, the JLO algorithm produces exactly the same local recursive calculations as the forward phase of the F-B algorithm.

One can also show an equivalence between the backward phase of the F-B algorithm and the JLO inference algorithm. Let the "leftmost" clique in the chain, $\left(H_{1}, H_{2}\right)$, be the root clique, and define a schedule such that the flows go from right to left. Figure 13 shows a local portion of the clique tree and the associated flows. Consider that the potential on clique $\left(H_{i}, H_{i+1}\right)$ has been updated already by earlier flows from the right. Thus, by definition,

$$
f_{\Phi_{i+1, N}}^{*}\left(h_{i}, h_{i+1}\right)=p\left(h_{i}, h_{i+1}, \phi_{i+1, N}^{*}\right) .
$$

The potential on the separator between $\left(H_{i}, H_{i+1}\right)$ and $\left(H_{i-1}, H_{i}\right)$ is calculated as

$$
\begin{aligned}
f_{\Phi_{i+1, N}}^{*}\left(h_{i}\right) & =\sum_{h_{i+1}} p\left(h_{i}, h_{i+1}, \phi_{i+1, N}^{*}\right) \\
& =p\left(h_{i}\right) \sum_{h_{i+1}} p\left(h_{i+1} \mid h_{i}\right) p\left(o_{i+1}^{*} \mid h_{i+1}\right) p\left(\phi_{i+2, N}^{*} \mid h_{i+1}\right)
\end{aligned}
$$

(by virtue of the various conditional independence relations in $\operatorname{HMM}(1,1)$ )

$$
\begin{aligned}
& =p\left(h_{i}\right) \sum_{h_{i+1}} p\left(h_{i+1} \mid h_{i}\right) p\left(o_{i+1}^{*} \mid h_{i+1}\right) \frac{p\left(\phi_{i+2, N}^{*}, h_{i+1}\right)}{p\left(h_{i+1}\right)} \\
& =p\left(h_{i}\right) \sum_{h_{i+1}} p\left(h_{i} \mid h_{i+1}\right) p\left(o_{i+1}^{*} \mid h_{i+1}\right) \frac{f_{\Phi_{i+2, N}}^{*}\left(h_{i+1}\right)}{p\left(h_{i+1}\right)} .
\end{aligned}
$$

Defining the update factor on this separator yields

$$
\begin{aligned}
\lambda_{\Phi_{i+1, N}}^{*}\left(h_{i}\right) & =\frac{f_{\Phi_{i+2, N}}^{*}\left(h_{i}\right)}{p\left(h_{i}\right)} \\
& =\sum_{h_{i+1}} p\left(h_{i} \mid h_{i+1}\right) p\left(o_{i+1}^{*} \mid h_{i+1}\right) \frac{f_{\Phi_{i+2, N}}^{*}\left(h_{i+1}\right)}{p\left(h_{i+1}\right)} \\
& =\sum_{h_{i+1}} p\left(h_{i} \mid h_{i+1}\right) p\left(o_{i+1}^{*} \mid h_{i+1}\right) \lambda_{\Phi_{i+2, N}^{*}}^{*}\left(h_{i+1}\right) .
\end{aligned}
$$




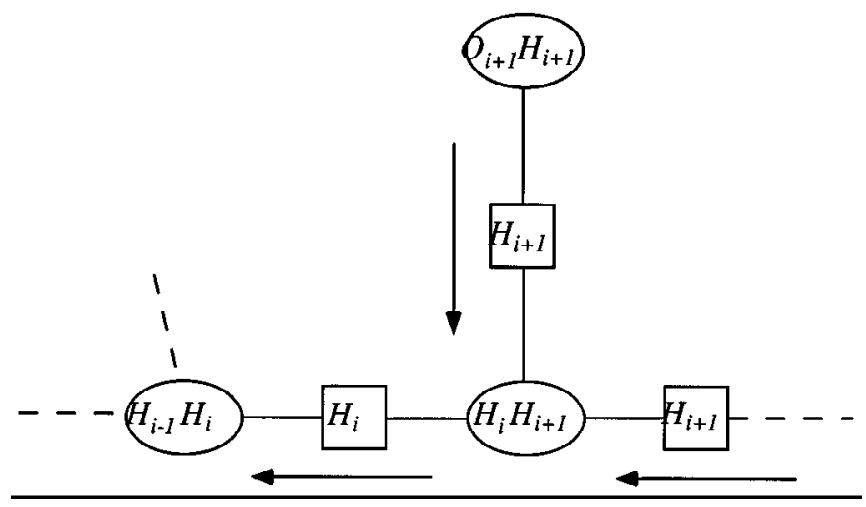

Figure 13: Local message passing in the $\operatorname{HMM}(1,1)$ junction tree during the collect phase of a right-to-left schedule. Ovals indicate cliques, boxes indicate separators, and arrows indicate flows.

This set of recursive equations in $\lambda$ corresponds exactly to the recursive equation (equation 25 in Rabiner 1989) for the $\beta$ variables in the backward phase of the F-B algorithm. In fact, the update factors $\lambda$ on the separators are exactly the $\beta$ variables. Thus, we have shown that the JLO inference algorithm recreates the F-B algorithm for the special case of the $\operatorname{HMM}(1,1)$ probability model.

Appendix B: The Viterbi Algorithm for $\operatorname{HMM(1,1)}$ Is a Special Case of Dawid's Algorithm. As with the inference problem, let the final clique in the chain containing $\left(H_{N-1}, H_{N}\right)$ be the root clique and use the same schedule: first a left-to-right collection phase into the root clique, followed by a right-to-left distribution phase out from the root clique. Again it is assumed that the junction tree has been initialized so that the potential functions are the local marginals, and the observable evidence $e$ has been entered into the cliques in the same manner as described for the inference algorithm.

We refer again to Figure 12. The sequence of flow and absorption operations is identical to that of the inference algorithm with the exception that marginalization operations are replaced by maximization. Thus, the potential on the separator between $\left(\mathrm{O}_{i}, H_{i}\right)$ and $\left(H_{i-1}, H_{i}\right)$ is initially updated to

$$
\hat{f}_{O_{i}}\left(h_{i}\right)=\max _{o_{i}} p\left(h_{i}, o_{i}\right)=p\left(h_{i}, o_{i}^{*}\right)
$$


The update factor for this separator is

$$
\lambda_{O_{i}}\left(h_{i}\right)=\frac{p\left(h_{i}, o_{i}^{*}\right)}{p\left(h_{i}\right)}=p\left(o_{i}^{*} \mid h_{i}\right),
$$

and after absorption into the clique $\left(H_{i-1}, H_{i}\right)$ one gets

$$
\hat{f}_{O_{i}}\left(h_{i-1}, h_{i}\right)=p\left(h_{i-1}, h_{i}\right) p\left(o_{i}^{*} \mid h_{i}\right)
$$

Now consider the flow from clique $\left(H_{i-2}, H_{i-1}\right)$ to $\left(H_{i-1}, H_{i}\right)$. Let $H_{i, j}=$ $\left\{H_{i}, \ldots, H_{j}\right\}$ denote a set of consecutive observable variables and $h_{i, j}^{*}=$ $\left\{h_{i}^{*}, \ldots, h_{j}^{*}\right\}$, denote the observed values for these variables, $1 \leq i<j \leq N$. Assume that the potential on separator $H_{i-1}$ has been updated to

$$
\hat{f}_{\Phi_{1, i-1}}\left(h_{i-1}\right)=\max _{h_{1, i-2}} p\left(h_{i-1}, h_{1, i-2}, \phi_{1, i-1}^{*}\right)
$$

by earlier flows in the schedule. Thus, the update factor for separator $H_{i-1}$ becomes

$$
\lambda_{\Phi_{1, i-1}}\left(h_{i-1}\right)=\frac{\max _{h_{1, i-2}} p\left(h_{i-1}, h_{1, i-2}, \phi_{1, i-1}^{*}\right)}{p\left(h_{i-1}\right)},
$$

and this gets absorbed into clique $\left(H_{i-1}, H_{i}\right)$ to produce

$$
\begin{aligned}
\hat{f}_{\Phi_{1, i}}\left(h_{i-1}, h_{i}\right) & =\hat{f}_{O_{i}}\left(h_{i-1}, h_{i}\right) \lambda_{\Phi_{1, i-1}}\left(h_{i-1}\right) \\
& =p\left(h_{i-1}, h_{i}\right) p\left(o_{i}^{*} \mid h_{i}\right) \frac{\max _{h_{1, i-2}} p\left(h_{i-1}, h_{1, i-2}, \phi_{1, i-1}^{*}\right)}{p\left(h_{i-1}\right)} .
\end{aligned}
$$

We can now obtain the new potential on the separator for the flow from clique $\left(H_{i-1}, H_{i}\right)$ to $\left(H_{i}, H_{i+1}\right)$,

$$
\begin{aligned}
\hat{f}_{\Phi_{1, i}}\left(h_{i}\right) & =\max _{h_{i-1}} \hat{f}_{\Phi_{1, i}}\left(h_{i-1}, h_{i}\right) \\
& =p\left(o_{i}^{*} \mid h_{i}\right) \max _{h_{i-1}}\left\{p\left(h_{i} \mid h_{i-1}\right) \max _{h_{1, i-2}} p\left(h_{i-1}, h_{1, i-2}, \phi_{1, i-1}^{*}\right)\right\} \\
& =p\left(o_{i}^{*} \mid h_{i}\right) \max _{h_{1, i-1}}\left\{p\left(h_{i} \mid h_{i-1}\right) p\left(h_{i-1}, h_{1, i-2}, \phi_{1, i-1}^{*}\right)\right\} \\
& =\max _{h_{1, i-1}} p\left(h_{i}, h_{1, i-1}, \phi_{1, i}^{*}\right),
\end{aligned}
$$

which is the result one expects for the updated potential at this clique. Thus, we can express the separator potential $\hat{f}_{\Phi_{1, i}}\left(h_{i}\right)$ recursively (via equation B.10) as

$$
\hat{f}_{\Phi_{1, i}}\left(h_{i}\right)=p\left(o_{i}^{*} \mid h_{i}\right) \max _{h_{i-1}}\left\{p\left(h_{i} \mid h_{i-1}\right) \hat{f}_{\Phi_{1, i-1}}\left(h_{i-1}\right)\right\} .
$$


This is the same recursive equation as used in the $\delta$ variables in the Viterbi algorithm (equation 33a in Rabiner 1989): the separator potentials in Dawid's algorithm using a left-to-right schedule are exactly the same as the $\delta^{\prime}$ s used in the Viterbi method for solving the MAP problem in $\operatorname{HMM}(1,1)$.

Proceeding recursively in this manner, one finally obtains at the root clique

$$
\hat{f}_{\Phi_{1, N}}\left(h_{N-1}, h_{N}\right)=\max _{h_{1, N-2}} p\left(h_{N-1}, h_{N}, h_{N-2}, \phi_{1, N}^{*}\right)
$$

from which one can get the likelihood of the evidence given the most likely state of the hidden variables:

$$
\begin{aligned}
\hat{f}(e) & =\max _{h_{N-1}, h_{N}} \hat{f}_{\Phi_{1, N}}\left(h_{N-1}, h_{N}\right) \\
& =\max _{h_{1, N}} p\left(h_{1, N}, \phi_{1, N}^{*}\right) .
\end{aligned}
$$

Identification of the values of the hidden variables that maximize the evidence likelihood can be carried out in the standard manner as in the Viterbi method, namely, by keeping a pointer at each clique along the flow in the forward direction back to the previous clique and then backtracking along this list of pointers from the root clique after the collection phase is complete. An alternative approach is to use the distribute phase of the Dawid algorithm. This has the same effect: Once the distribution flows are completed, each local clique can calculate both the maximum value of the evidence likelihood given the hidden variables and the values of the hidden variables in this maximum that are local to that particular clique.

\section{Acknowledgments}

MIJ gratefully acknowledges discussions with Steffen Lauritzen on the application of the IPF algorithm to UPINs. The research described in this article was carried out in part by the Jet Propulsion Laboratory, California Institute of Technology, under a contract with the National Aeronautics and Space Administration.

\section{References}

Baum, L. E., and Petrie, T. 1966. Statistical inference for probabilistic functions of finite state Markov chains. Ann. Math. Stat. 37, 1554-1563.

Bishop, Y. M. M., Fienberg, S. E., and Holland, P. W. 1973. Discrete Multivariate Analysis: Theory and Practice. MIT Press, Cambridge, MA.

Buntine, W. 1994. Operations for learning with graphical models. Journal of Artificial Intelligence Research 2, 159-225.

Buntine, W. In press. A guide to the literature on learning probabilistic networks from data. IEEE Transactions on Knowledge and Data Engineering. 
Dawid, A. P. 1992a. Applications of a general propagation algorithm for probabilistic expert systems. Statistics and Computing 2, 25-36.

Dawid, A. P. 1992b. Prequential analysis, stochastic complexity, and Bayesian inference (with discussion). In Bayesian Statistics 4, J. M. Bernardo, J. Berger, A. P. Dawid, and A. F. M. Smith, eds., pp. 109-125. Oxford University Press, London.

DeGroot, M. 1970. Optimal Statistical Decisions. McGraw-Hill, New York.

Dempster, A., Laird, N., and Rubin, D. 1977. Maximum likelihood from incomplete data via the EM algorithm. Journal of the Royal Statistical Society, Series $B$ 39, 1-38.

Elliott, R. J., Aggoun, L., and Moore, J. B. 1995. Hidden Markov Models: Estimation and Control. Springer-Verlag, New York.

Frasconi, P., and Bengio, Y. 1994. An EM approach to grammatical inference: Input/output HMMs. In Proceedings of the 12th IAPR Intl. Conf. on Pattern Recognition, pp. 289-294. IEEE Computer Society Press, Los Altimos, CA.

Gauvain, J., and Lee, C. 1994. Maximum a posteriori estimation for multivariate Gaussian mixture observations of Markov chains. IEEE Trans. Sig. Audio Proc. 2, 291-298.

Geman, S., and Geman, D. 1984. Stochastic relaxation, Gibbs distributions, and the Bayesian restoration of images. IEEE Trans. Patt. Anal. Mach. Intell. 6, 721-741.

Ghahramani, Z., and Jordan, M. I. 1996. Factorial hidden Markov models. In Advances in Neural Information Processing Systems 8, D. S. Touretzky, M. C. Mozer, and M. E. Hasselmo, eds., pp. 472-478. MIT Press, Cambridge, MA.

Heckerman, D., and Geiger, D. 1995. Likelihoods and Priors for Bayesian Networks. MSR-TR-95-54. Microsoft Corporation, Redmond, WA.

Heckerman, D., Geiger, D., and Chickering, D. 1995. Learning Bayesian networks: The combination of knowledge and statistical data. Machine Learning 20, 197-243.

Hinton, G. E., and Sejnowski, T. J. 1986. Learning and relearning in Boltzmann machines. In Parallel Distributed Processing: Explorations in the Microstructure of Cognition, D. E. Rumelhart, J. L. McClelland, and the PDP Research Group, eds., vol. 1, chap. 7. MIT Press, Cambridge, MA.

Huang, X. D., Ariki, Y., and Jack, M. A. 1990. Hidden Markov Models for Speech Recognition. Edinburgh University Press, Edinburgh.

Isham, V. 1981. An introduction to spatial point processes and Markov random fields. International Statistical Review 49, 21-43.

Itzykson, C., and Drouffé, J-M. 1991. Statistical Field Theory. Cambridge University Press, Cambridge.

Jensen, F. V., Lauritzen, S. L., and Olesen, K. G. 1990. Bayesian updating in recursive graphical models by local computations. Computational Statistical Quarterly 4, 269-282.

Jiřousek, R., and Přeučil, S. 1995. On the effective implementation of the iterative proportional fitting procedure. Computational Statistics and Data Analysis 19, 177-189. 
Kass, R., Tierney, L., and Kadane, J. 1988. Asymptotics in Bayesian computation. In Bayesian Statistics 3, J. Bernardo, M. DeGroot, D. Lindley, and A. Smith, eds., pp. 261-278. Oxford University Press, Oxford.

Kent, R. D., and Minifie, F. D. 1977. Coarticulation in recent speech production models. Journal of Phonetics 5, 115-117.

Lauritzen, S. L., and Spiegelhalter, D. J. 1988. Local computations with probabilities on graphical structures and their application to expert systems (with discussion). J. Roy. Statist. Soc. Ser. B. 50, 157-224.

Lauritzen, S. L., Dawid, A. P., Larsen, B. N., and Leimer, H. G. 1990. Independence properties of directed Markov fields. Networks 20, 491-505.

Lauritzen, S., and Wermuth, N. 1989. Graphical models for associations between variables, some of which are qualitative and some quantitative. Annals of Statistics 17, 31-57.

Lindblom, B. 1990. Explaining phonetic variation: A sketch of the H\&H theory. In Speech Production and Speech Modeling, W. J. Hardcastle and A. Marchal, eds., pp. 403-440. Kluwer, Dordrecht.

Lucke, H. 1995. Bayesian belief networks as a tool for stochastic parsing. Speech Communication 16, 89-118.

MacKay, D. J. C. 1992a. Bayesian interpolation. Neural Computation 4, 415-447.

MacKay, D. J. C. 1992b. A practical Bayesian framework for backpropagation networks. Neural Computation 4, 448-472.

Madigan, D., and Raftery, A. E. 1994. Model selection and accounting for model uncertainty in graphical models using Occam's window. J. Am. Stat. Assoc. 89, 1535-1546.

Modestino, J., and Zhang, J. 1992. A Markov random field model-based approach to image segmentation. IEEE Trans. Patt. Anal. Mach. Int. 14(6), 606615.

Morgenstern, I., and Binder, K. 1983. Magnetic correlations in two-dimensional spin-glasses. Physical Review B 28, 5216.

Neal, R. 1993. Probabilistic inference using Markov chain Monte Carlo methods. CRGTR-93-1. Department of Computer Science, University of Toronto.

Pearl, J. 1988. Probabilistic Reasoning in Intelligent Systems: Networks of Plausible Inference. Morgan Kaufmann, San Mateo, CA.

Pearl, J., Geiger, D., and Verma, T. 1990. The logic of influence diagrams. In Influence Diagrams, Belief Nets, and Decision Analysis, R. M. Oliver and J. Q. Smith, eds., pp. 67-83. John Wiley, Chichester, UK.

Perkell, J. S., Matthies, M. L., Svirsky, M. A., and Jordan, M. I. 1993. Trading relations between tongue-body raising and lip rounding in production of the vowel /u/: A pilot motor equivalence study. Journal of the Acoustical Society of America 93, 2948-2961.

Poritz, A. M. 1988. Hidden Markov models: A guided tour. In Proceedings of the IEEE International Conference on Acoustics, Speech and Signal Processing, 1:7-13, IEEE Press, New York.

Rabiner, L. 1989. A tutorial on hidden Markov models and selected applications in speech recognition. Proceedings of the IEEE 77, 257-285. 
Raftery, A. 1995. Bayesian model selection in social research (with discussion). In Sociological Methodology, P. Marsden, ed., pp. 111-196. Blackwell, Cambridge, MA.

Rissanen, J. 1987. Stochastic complexity (with discussion). Journal of the Royal Statistical Society, Series B 49, 223-239, 253-265.

Saul, L. K., and Jordan, M. I. 1995. Boltzmann chains and hidden Markov models. In Advances in Neural Information Processing Systems 7, G. Tesauro, D. S. Touretzky, and T. K. Leen, eds., pp. 435-442. MIT Press, Cambridge, MA.

Saul, L. K., and Jordan, M. I. 1996. Exploiting tractable substructures in intractable networks. In Advances in Neural Information Processing Systems 8, D. S. Touretzky, M. C. Mozer, and M. E. Hasselmo, eds., pp. 486-492. MIT Press, Cambridge, MA.

Schwarz, G. 1978. Estimating the dimension of a model. Annals of Statistics 6, 461-464.

Shachter, R. D., Anderson, S. K., and Szolovits, P. 1994. Global conditioning for probabilistic inference in belief networks. In Proceedings of the Uncertainty in AI Conference 1994, pp. 514-522. Morgan Kaufmann, San Mateo, CA.

Spiegelhalter, D. J., Dawid, A. P., Hutchinson, T. A., and Cowell, R. G. 1991. Probabilistic expert systems and graphical modelling: A case study in drug safety. Phil. Trans. R. Soc. Lond. A 337, 387-405.

Spiegelhalter, D. J., and Lauritzen, S. L. 1990. Sequential updating of conditional probabilities on directed graphical structures. Networks 20, 579-605.

Spirtes, P., and Meek, C. 1995. Learning Bayesian networks with discrete variables from data. In Proceedings of First International Conference on Knowledge Discovery and Data Mining, pp. 294-299. AAAI Press, Menlo Park, CA.

Stolorz, P. 1994. Recursive approaches to the statistical physics of lattice proteins. In Proc. 27th Hawaii Intl. Conf. on System Sciences, L. Hunter, ed., 5:316-325.

Swendsen, R. H., and Wang, J-S. 1987. Nonuniversal critical dynamics in Monte Carlo simulations. Physical Review Letters 58.

Thiesson, B. 1995. Score and information for recursive exponential models with incomplete data. Tech. rep. Institute of Electronic Systems, Aalborg University, Aalborg, Denmark.

Vandermeulen, D., Verbeeck, R., Berben, L., Delaere, D., Suetens, P., and Marchal, G. 1994. Continuous voxel classification by stochastic relaxation: Theory and application to MR imaging and MR angiography. Image and Vision Computing 12(9), 559-572.

Whittaker, J. 1990. Graphical Models in Applied Multivariate Statistics. John Wiley, Chichester, UK.

Williams, C., and Hinton, G. E. 1990. Mean field networks that learn to discriminate temporally distorted strings. In Proc. Connectionist Models Summer School, pp. 18-22. Morgan Kaufmann, San Mateo, CA.

Received February 2, 1996; accepted April 22, 1996. 